\title{
Effects of Household Storage and Plumbing Systems on the Levels of Trace Elements in Desalinated Drinking Water in Kuwait
}

\author{
Humood F. Al-Mudhaf' ${ }^{1}$, Nabeel M. Al-Khulaifi', Mohammad N. Al-Hayan", \\ Abdel-Sattar I. Abu-Shady ${ }^{1 *}$ \\ ${ }^{1}$ Chemical Engineering Technology Department, College of Technological Studies, Public Authority for Applied Education and \\ Training, Adailiyah, Kuwait; ${ }^{2}$ Environmental Health Department, College of Health Sciences, Public Authority for Applied Educa- \\ tion and Training, Adailiyah, Kuwait. \\ Email: *aasattar44@yahoo.com
}

Received June $9^{\text {th }}, 2012$; revised August $1^{\text {st }}, 2012$; accepted September $2^{\text {nd }}, 2012$

\begin{abstract}
Household desalinated drinking water samples collected from outdoor points and from indoor consumption points at 99 locations representing more than $95 \%$ of the residential areas in Kuwait were analyzed for 25 trace elements and water quality parameters. Only $\mathrm{Al}, \mathrm{Cr}, \mathrm{Co}, \mathrm{Cu}, \mathrm{Fe}, \mathrm{Pb}, \mathrm{Ni}$, and $\mathrm{Zn}$ were found to be over-represented at the consumption point compared with the outdoor point, with wide variations among the sampling locations and elements. The highest increases were observed for Fe (135\%) and $\mathrm{Zn}(123 \%)$, followed by $\mathrm{Pb}(69 \%), \mathrm{Co}(58 \%), \mathrm{Cu}(42 \%), \mathrm{Cr}(31 \%)$, and $\mathrm{Al}$ $(30 \%)$, and the lowest increase was observed for $\mathrm{Ni}(19 \%)$. In most cases, the increases in $\mathrm{Cu}, \mathrm{Fe}$, and $\mathrm{Zn}$ were inversely proportional to the conductivity and directly proportional to the $\mathrm{Cl}^{-}$concentration. In the outdoor samples, only Fe exceeded the US-EPA guideline (in 3\% of the outdoor samples taken), whereas $\mathrm{Fe}, \mathrm{Pb}$, and $\mathrm{Ni}$ exceeded the US-EPA and WHO guidelines in $8.5 \%, 0.3 \%$, and $1 \%$ of the indoor consumption point samples, respectively. Thus, leaching from household utilities may cause health concerns for consumers of drinking water in Kuwait. The increases in Fe were the highest in the summer (240\%), and in this regard, Fe exhibited the greatest difference between summer and winter (the increase was $139 \%$ higher in the summer). The results of the present study may be useful for water production authorities and consumers in Kuwait and suggest the use of alternative new pipes with more resistant internal coatings and connecting techniques.
\end{abstract}

Keywords: Desalinated Water; Kuwait; Corrosion; Leaching; Trace Elements

\section{Introduction}

Of all of the countries of the world, particularly the Arab Gulf countries, Kuwait has the most limited fresh-water resources and the lowest levels of renewable fresh water [1]. The desalination of sea water provides the only source of drinking water in Kuwait, and the amount of desalination required increases as the demand for potable water increases. To produce distilled water, multi-stage flash (MSF) distillation is used by desalination plants in Kuwait. The distilled water produced is saturated with calcium carbonate and remineralized by blending it with $5 \%-10 \%$ brackish water to make it potable. Finally, the remineralized water is chlorinated and pumped to a distribution network. The remineralization process prevents or limits corrosion within the distribution network's pipes and alters the trace elements (TEs) present and the

${ }^{*}$ Corresponding author. mineral composition in the desalinated drinking water relative to that produced from surface and ground freshwater sources. In addition, the saturation of the remineralized water with calcium carbonate provides a stable passivation layer that further prevents the corrosion of transfer pipes [2-4]. However, the World Health Organization (WHO) has reported that cardiovascular disease can develop when remineralized desalinated drinking water is consumed [5].

A literature survey indicated that numerous studies have been performed worldwide to evaluate the TEs in drinking water produced from ground or surface water $[4$, 6-9] or through the thermal desalination of seawater [1014]. Desalinated household drinking water (HW) in $\mathrm{Ku}-$ wait has been extensively studied to evaluate the levels of TEs [12,15], minerals [16], disinfection byproducts (DBPs) known as haloacetic acids [17,18] and halomethanes [19,21-23], and organic contaminants [24]. 
Moreover, Al-Fraij et al. reviewed the various sources of contamination of drinking water in Kuwait during the transportation to consumers [25] and concluded that the monitoring process is inadequate. Al-Mudahf and AbuShady [15] reported an increase in the levels of some TEs in HW at the consumption point compared with the results of a previous study by Al-Fraij et al. [12] on samples collected at the reservoirs at desalination plants before pumping to the distribution network. There is a worldwide interest in studying the effects of the types and compositions of distribution systems and household utilities on the contamination of drinking water by TEs through the leaching and migration of corrosion products [26-34]. The major sources of indoor TE pollution include brass faucets and fixtures and household plumbing lines and their solders [28,30,32]. Viraraghavan et al. [32] reviewed the impact of the migration of some metals from household plumbing systems on the quality of drinking water. Alam and Sadiq [33] reported an increase in the levels of copper, iron, and zinc in desalinated drinking water in Dhahran, Saudi Arabia, mediated by the transportation to the consumers. Recently, Veschetti et al. [26] studied the migration of trace metals from distribution networks into Italian drinking water and related the high levels of Ni outliers to tap materials, Fe to corrosion processes, and $\mathrm{Pb}$ to lead pipes that still existed in old buildings.

In Kuwait, consumers drink water that has passed from the outdoor point of the public distribution system to their taps via their private household plumbing, piping, and storage utilities. The TE content and quality of this water may have changed during its residence and transport through these utilities. The main objectives of this study were 1) to determine the contamination of $\mathrm{HW}$ at indoor consumption points by TEs as a result of leaching from the metallic materials of household utilities; 2) to explore the impacts of various household storage and plumbing systems on the levels of TEs and the quality of HW at consumption points; 3 ) to correlate the variation in TE content to water composition; and 4) to study seasonal variations in TE levels.

\section{Materials and Methods}

\subsection{Reagents and Reference Materials}

For all of the required preparations, we used ultra-pure Type I ICP/MS-grade inorganic-free water obtained from a Millipore Ultrapure Water Purification System equipped with a Milli-Q element, a Q-Guard II pack, a Quantum VX cartridge, a $0.22-\mu \mathrm{m}$ Millipak filter, and a $<0.1-\mu \mathrm{m}$ optimizer filter at the point of use (Millipore, Bedford, MA, USA). Certified high-purity chemical reagents, individual and calibration standard mixes, and quality con- trol and reference materials were obtained from Agilent Technologies (Santa Clara, CA, USA), AccuStandard (New Haven, CT, USA), SPEX CertiPrep, Inc. (Metuchen, NJ, USA), Alfa Aesar (Ward Hill, MA, USA), and Merck (Nottingham, UK).

\subsection{Desalination Plants and Sampling}

According to official information from the Ministry of Electricity and Water in Kuwait (MEW), five dual-purpose power and desalination plants located along the Kuwaiti coast used MSF distillation to produce a total of >310 million imperial gallons per day (MIGD) of distillate (yielding 331.5 MIGD of potable water) during the course of this study. To prevent marine fouling inside the distillers, the seawater supply at each plant (a total of $\sim 18.7 \mathrm{~mm}^{3} \cdot \mathrm{d}^{-1}$ for the five plants) was chlorinated $(\sim 6.0$ $\mathrm{mg} \cdot \mathrm{L}^{-1}$ total residual chlorine); this seawater was used to cool and feed the MSF distillation units. The $\mathrm{pH}$ of the distilled water that was produced was adjusted to 8.3 by carbonation, and the water was blended with $5 \%-10 \%$ brackish water followed by chlorination to a total residual chlorine value of $\sim 1.2 \mathrm{mg} \cdot \mathrm{L}^{-1}$. The chlorinated water was pumped first to storage facilities (towers and concrete underground reservoirs) and then to the distribution system network and ultimately to consumer storage facilities. The Az-Zoor plant (with a mean capacity of 115.2 MIGD) completely supplies the Umm Al-Haiman (DUH) location. Together with the Shuaiba plant (36 MIGD), the Az-Zoor plant supplies all of the locations in the Ahmadi Governorate (GAH) and the Mubarak Al-Kabeer Governorate (GMK) with various capacity ratios. The Doha West (110.4 MIGD) and Doha East (50.4 MIGD) plants primarily supply the Shuwaikh Education (CSE) location and all of the residential areas of the Jahra Governorate (GJA). The Shuwaikh (19.5 MIGD) plant primarily supplies the Shuwaikh Sakani (CSW) location. All of the other individual locations in the residential areas of the Capital (GCA), Farwaniya (GFA), and Hawalli (GHA) governorates are supplied by all of the plants at varying ratios depending on temporal demand. The main distribution piping network was constructed from asbestos/ cement or from galvanized iron pipes, and most of this piping was recently replaced with ductile iron pipes. Figure 1 shows both the locations of the desalination plants and the distribution of the sampling points within the locations in the various governorates. Three replicates of each sample were collected and preserved according to US-EPA Methods 300.1 and 200.8 [35]. The samples were delivered to the laboratory in boxes that were cooled with dry ice and were refrigerated at $4^{\circ} \mathrm{C}$ until analysis. All of the water samples were analyzed within the recommended holding time [35] or discarded. 


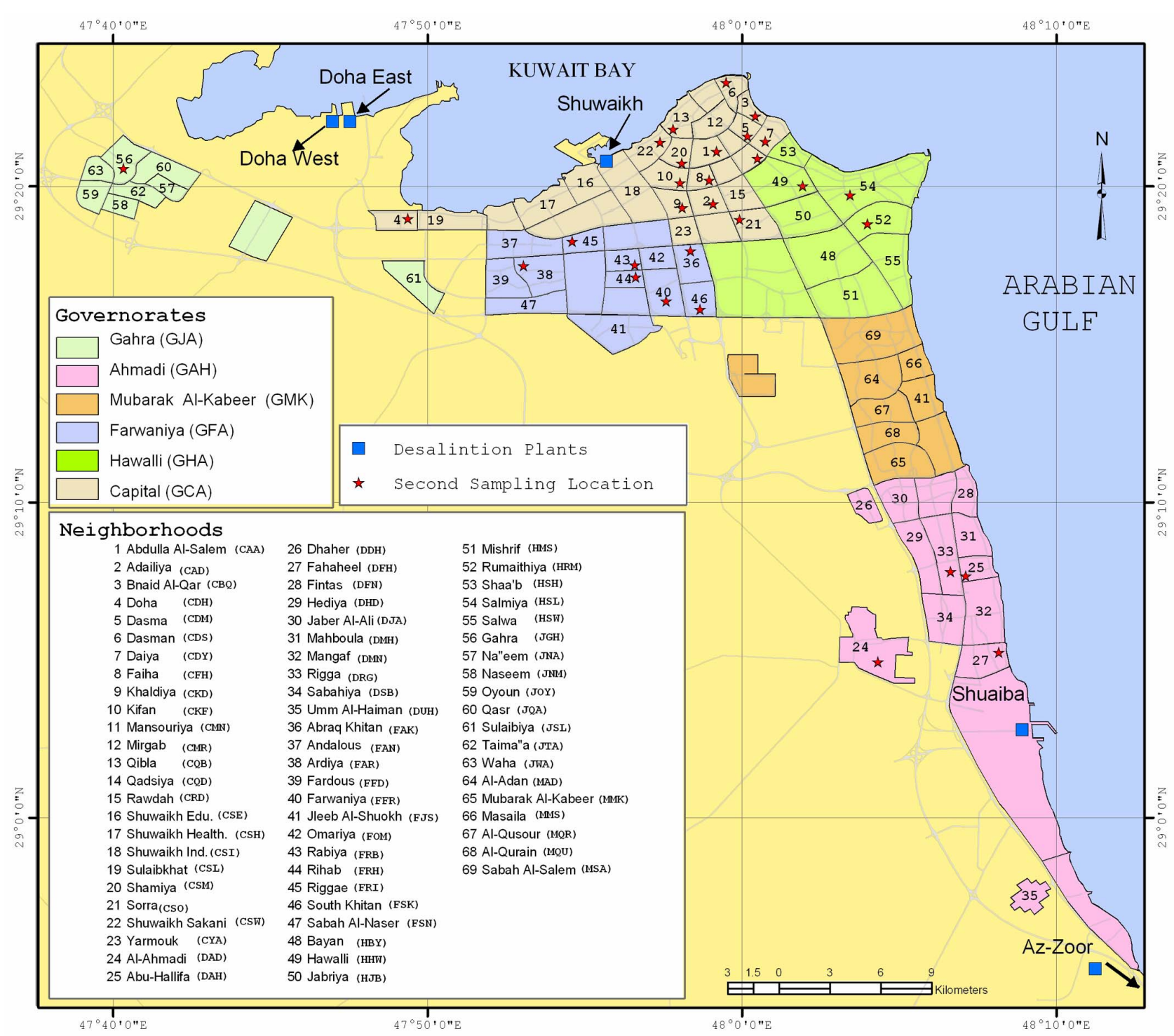

Figure 1. Locations of the desalination plants and the distributions of sampling points within the different neighborhoods of the governorates of Kuwait.

\subsection{Determination of Analyte Concentrations}

The temperature, $\mathrm{pH}$, electrical conductivity (EC), total dissolved solids (TDS), and residual chlorine (Cl) were measured on-site at the time of collection. Inorganic anion concentrations were determined according to USEPA 300.1 method [35] using a Waters gradient HPLC system (Waters, Milford, MA, USA) equipped with a Waters 432 conductivity detector, an Altech 1000 HP Autosuppressor, and a Waters 2690 Separations Module. The levels of $25 \mathrm{TEs}$, i.e., aluminum (Al), arsenic (As), boron $(\mathrm{B})$, barium $(\mathrm{Ba})$, beryllium $(\mathrm{Be})$, cadmium $(\mathrm{Cd})$, calcium $(\mathrm{Ca})$, chromium $(\mathrm{Cr})$, cobalt $(\mathrm{Co})$, copper $(\mathrm{Cu})$, iron $(\mathrm{Fe})$, lead $(\mathrm{Pb})$, magnesium $(\mathrm{Mg})$, manganese $(\mathrm{Mn})$, mercury $(\mathrm{Hg})$, molybdinum $(\mathrm{Mo})$, nickel $(\mathrm{Ni})$, potassium
$(\mathrm{K})$, antimony $(\mathrm{Sb})$, selenium ( $\mathrm{Se})$, sodium $(\mathrm{Na})$, strontium (Sr), titanium (Ti), vanadium $(\mathrm{V})$, and zinc $(\mathrm{Zn})$, were measured according to US-EPA Method 200.8 [35] with an Agilent ICP/MS 7500ce (Agilent, Palo Alto, CA, USA) that was equipped with an octopole reaction system, a micromist nebulizer, a bonnet and shield, and an integrated autosampler. Details regarding the analytical procedures, quality control (QC), quality assurance (QA), and detection limits (DLs) have been reported elsewhere $[15,16]$.

\section{Results and Discussion}

HW samples were collected from private residences (houses and apartments) and from offices and public 
buildings after flushing for five minutes. Ninety-nine locations were sampled in 69 neighborhoods in the six governorates of Kuwait. Together, these locations cover more than $95 \%$ of the residential areas in Kuwait. At each sampling collection, two samples were collected from two points in each selected building on each occasion: one from the water connection at the point of the distribution network just outside the building (outdoor$\mathrm{HW}$; designated as O-HW) and the second from an inside faucet (tap) after the drinking water had passed through the storage and plumbing facilities as well as the household piping system of the building (indoor-HW; designated as I-HW). At residential or public properties, the storage tanks are mainly roof-top tanks $\left(6-12 \mathrm{~m}^{3}\right)$ that are constructed of galvanized iron. These iron tanks are generally being replaced with fiberglass, although ground reservoirs constructed from the same materials (20 - 100 $\mathrm{m}^{3}$ ) are also used for large buildings. All of the available information on the household storage, piping, and other utilities in the studied premises is given in Table 1, as are the types of outdoor distribution network piping in each area.

Three hundred and twenty-one I-HW samples and the same number of O-HW samples were collected simultaneously between December 2003 and May 2005 with different sampling frequencies (ranging from 1 to 20 sampling events) from 16, 38, 19, 11, 9, and 6 different sampling locations within the residential areas of the GAH, GCA, GFA, GHA, GJA, and GMK governorates, respectively (Figure 1). To achieve the objectives of the study, 12 subareas of higher population density within the 99 sampling locations in the 69 neighborhoods of the governorates were identified and investigated using a higher sampling frequency. Accordingly, 6 - 20 samples were periodically collected from each of the Kifan (CKF), Qibla (CQB), Shuwaikh education (CSE), Shuwaikh Health (CSH), Sulaibekhat (CSL), Sorra (CSO), CSW, DUH, Ardiya (FAR), Riggae (FRI), Salmiya (HSL), and Oyoon (JOY) locations, as indicated in Figure 1. At each of the remaining 87 locations, 1 - 3 samples were collected.

The corrosion of construction products that contain metallic materials and contact drinking water (CP-DW) during its production and transportation via the general distribution network and through private household utilities represents a major health concern because of the

Table 1. Types of household storage, piping, and other utilities at various sampling points.

\begin{tabular}{|c|c|c|c|c|c|c|c|}
\hline \multirow{2}{*}{ S. No. } & \multirow{2}{*}{$\begin{array}{c}\text { Sampling } \\
\text { location/type }\end{array}$} & \multirow{2}{*}{ Construction year } & \multirow{2}{*}{ Storage tank } & \multicolumn{2}{|c|}{ Indoor piping /length (m) } & \multirow{2}{*}{ Faucets } & \multirow{2}{*}{$\begin{array}{l}\text { Outdoor } \\
\text { piping }^{c}\end{array}$} \\
\hline & & & & $A^{\mathrm{a}}$ & $B^{\mathbf{b}}$ & & \\
\hline 1 & CKF/House ${ }^{d}$ & 1988 & Fiberglass & $\mathrm{GI} / 30$ & PVC./20 & $\mathrm{Ni} / \mathrm{Cr}$ & DI \\
\hline 2 & CQB/PB & 1957 & GI & $\mathrm{GI} / 35$ & $\mathrm{GI} / 25$ & copper & GI \\
\hline 3 & CSE/College & 2002 & Fiberglass & $\mathrm{PVC} / 150$ & $\mathrm{PVC} / 100$ & $\mathrm{Ni} / \mathrm{Cr}$ & DI \\
\hline 4 & CSH/Hospital & 1959 & GI & $\mathrm{GI} / 100$ & $\begin{array}{l}\mathrm{GI} / 40 \text { and } \\
\text { Copper/15 }\end{array}$ & $\mathrm{Ni} / \mathrm{Cr}$ & DI \\
\hline 5 & CSL/PB & 1984 & Fiberglass & $\mathrm{GI} / 65$ & $\mathrm{GI} / 50$ & $\mathrm{Ni} / \mathrm{Cr}$ & DI \\
\hline 6 & $\mathrm{CSO} / H o u s e^{\mathrm{d}}$ & 1972 & GI & $\mathrm{GI} / 45$ & $\begin{array}{l}\mathrm{GI} / 25 \text { and } \\
\text { Copper/20 }\end{array}$ & $\mathrm{Ni} / \mathrm{Cr}$ & DI \\
\hline 7 & CSW/OB & 1994 & Fiberglass & $\mathrm{GI} / 125$ & G./100 & $\mathrm{Ni} / \mathrm{Cr}$ & DI \\
\hline 8 & DUH/PB & 2001 & Fiberglass & $\mathrm{PVC} / 70$ & $\mathrm{PVC} / 55$ & $\mathrm{Ni} / \mathrm{Cr}$ & DI \\
\hline 9 & FAR/House ${ }^{d}$ & 1983 & GI & GI/45 & Copper/35 & $\mathrm{Ni} / \mathrm{Cr}$ & D. \\
\hline 10 & FRI/Apt. ${ }^{e}$ & 1979 & Fiberglass & $\mathrm{PVC} / 75$ & $\mathrm{PVC} / 40$ & $\mathrm{Ni} / \mathrm{Cr}$ & DI \\
\hline 11 & HSL/Apt. ${ }^{\mathrm{f}}$ & 1986 & Fiberglass & $\mathrm{PVC} / 30$ & $\mathrm{PVC} / 20$ & $\mathrm{Ni} / \mathrm{Cr}$ & DI \\
\hline 12 & JOY/House ${ }^{d}$ & 1992 & Fiberglass & $\mathrm{PVC} / 25$ & $\mathrm{PVC} / 15$ & $\mathrm{Ni} / \mathrm{Cr}$ & DI \\
\hline 13 & $\begin{array}{l}\text { Remaining } \\
\text { locations }^{\mathrm{g}}\end{array}$ & $\begin{array}{l}\text { In total, } 87 \text { buildings } \\
2001 \text { and contain va }\end{array}$ & $\begin{array}{l}\text { hat were sample } \\
\text { pus types of hou }\end{array}$ & $\begin{array}{l}\text { ith low freq } \\
\text { ld storage } t\end{array}$ & $\begin{array}{l}\text { ere construc } \\
\text { ping, faucets }\end{array}$ & $\begin{array}{l}\text { ween the } \\
\text { utdoor pip }\end{array}$ & of 1965 and \\
\hline
\end{tabular}

${ }^{a}$ Length from the outdoor point to the roof storage tank; ${ }^{b}$ Length from roof storage tank to the indoor sampling faucet; ${ }^{\mathrm{c}}$ Piping type outside the building; ${ }^{\mathrm{d}}$ Single family home (6 - 15 residents); ${ }^{\mathrm{e}}$ Apartment on the fifth floor of a nine-story residential building complex (64 apartments); ${ }^{\mathrm{f}} \mathrm{Apartment}$ on the second floor of a

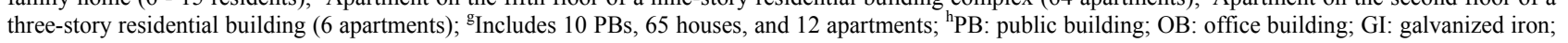
DI: ductile iron. 
leaching of the contaminants $\mathrm{Cd}, \mathrm{Cr}, \mathrm{Pb}$, and $\mathrm{Ni}$, which pose serious health threats to the consumers of drinking water [36]. Additionally, as a consequence of corrosion, other elements, including $\mathrm{Al}, \mathrm{Cu}, \mathrm{Fe}$, and $\mathrm{Zn}$, may leach into the drinking water. To protect the health of consumers of drinking water, the European Directive 98/83 on drinking water quality [37] established rules for monitoring these elements in CP-DW in the European Union. The interactions between the metallic materials of the CP-DW and water may be influenced by several factors that include the surface properties, metallic compositions, and lengths of the pipes as well as their surface/volume ratios, hydraulic conditions, network configurations, water residence times, and the physico-chemical characteristics of the bulk water $[38,39]$. The most corrosive waters are characterized by low $\mathrm{pH}$ levels, high carbon dioxide content, and low mineral content [40]. Each of these factors may affect the leaching of metals differently. In addition, the levels of TEs in the O-HW samples may be influenced, as previously desrcibed [16,17,22,24], by the operational processes that are used at the desalination plants, the nature of the intake seawater, the positions of the plants on the coast of Kuwait, and the nature and type of the distribution networks. In addition to these factors, the type of household storage and piping and plumbing facilities greatly affects the levels of some TEs at the consumer point (I-HW) [26,32,33]. It is also important to note that the sole source of HW in Kuwait is distilled water blended with brackish water. Thus, the differences found in the levels of TEs between O-HW and I-HW samples in various locations and governorates were also likely related to subtle variations in the characteristics of the brackish waters and the blend ratios used during the production process.

The 25 TEs analyzed in the 321 samples of O-HW collected in this study were $\mathrm{Al}, \mathrm{As}, \mathrm{B}, \mathrm{Ba}, \mathrm{Be}, \mathrm{Ca}, \mathrm{Cd}, \mathrm{Cr}$, $\mathrm{Co}, \mathrm{Cu}, \mathrm{Fe}, \mathrm{Pb}, \mathrm{Mg}, \mathrm{Mn}, \mathrm{Hg}, \mathrm{Mo}, \mathrm{Ni}, \mathrm{K} \mathrm{Sb}, \mathrm{Se}, \mathrm{Na}, \mathrm{Sr}$, $\mathrm{Ti}, \mathrm{V}$, and $\mathrm{Zn}$. In addition, we measured the water quality parameters (WQPs) $\mathrm{pH}$, conductivity, TDS, and residual $\mathrm{Cl}$ as well as the concentrations of the anions $\mathrm{Cl}^{-}$and $\mathrm{SO}_{4}^{2-}$. The levels of these TEs and the WQPs of the I$\mathrm{HW}$ as well as their variations among sampling locations and governorates have been presented and discussed previously $[15,16]$. Table 2 depicts the mean values of the TEs in the O-HW samples from various sampling locations and governorates of Kuwait, and the values of the I-HW samples are included for comparison.

The mean concentrations of $\mathrm{Al}, \mathrm{Cr}, \mathrm{Co}, \mathrm{Cu}, \mathrm{Fe}, \mathrm{Pb}, \mathrm{Ni}$, and $\mathrm{Zn}$ (designated as the 8-TE group) were higher in the I-HW samples than in the O-HW samples collected at all sampling locations. However, Be was not detected in any of the samples, and there were no significant differences among the mean values of the remaining 16 TEs in the
I-HW samples and those in the O-HW samples at various sampling locations (Table 2). The increases in the mean values of the concentrations of the elements in the 8-TE group in the I-HW samples compared with those in the O-HW samples were attributed mainly to leaching from household utilities and showed significant variations among sampling locations and among elements. Figure 2 illustrates the variations in the increases of the elements in the 8-TE group in all of the I-HW samples (321) collected from the 99 sampling locations in the six governorates in relation to those in the O-HW samples. The highest increases were found for $\mathrm{Fe}(135 \%)$ and $\mathrm{Zn}$ (123\%), followed by $\mathrm{Pb}(69 \%), \mathrm{Co}(58 \%), \mathrm{Cu}(42 \%), \mathrm{Cr}$ $(31 \%)$, and $\mathrm{Al}(30 \%)$, and $\mathrm{Ni}$ exhibited the lowest increase $(19 \%)$. There were wide variations in the increases for each of these TEs among the sampling locations and among the governorates, as shown in Figure 3.

Figure 3(a) shows that the highest and lowest increases of $\mathrm{Al}$ occurred in the I-HW samples of CQB (198\%) and CKF (3\%) among the locations and in the GCA (46\%) and GMK (9\%) samples, respectively, among the governorates. Table 2 shows that the highest mean $\mathrm{Al}$ values in the I-HW and O-HW samples (34.4 $\mu \mathrm{g} \cdot \mathrm{L}^{-1}$ and $33.5 \mu \mathrm{g} \cdot \mathrm{L}^{-1}$, with ranges of $21.2-53.0$ and $18.0-53.3 \mu \mathrm{g} \cdot \mathrm{L}^{-1}$, respectively) occurred at the CKF location, which had the lowest increase of $\mathrm{Al}(3 \%)$. By contrast, the CQB location, which had the highest increase of $\mathrm{Al}(198 \%)$, had significantly lower levels in the I-HW and O-HW samples (mean values of 13.4 and 4.5 $\mu \mathrm{g} \cdot \mathrm{L}^{-1}$ and ranges of $1.0-54.4$ and $0.3-10.0 \mu \mathrm{g} \cdot \mathrm{L}^{-1}$, respectively). Thus, the migration of $\mathrm{Al}$ from household utilities and piping was the only source of $\mathrm{Al}$ increase in the I-HW samples collected at the CQB location, which was the location with the highest $\mathrm{Al}$ increase. In contrast, no Al leaching from household utilities and piping occurred at the CKF location, despite its high mean I-HW value $\left(34.4 \mu \mathrm{g} \cdot \mathrm{L}^{-1}\right)$, which was slightly higher than the

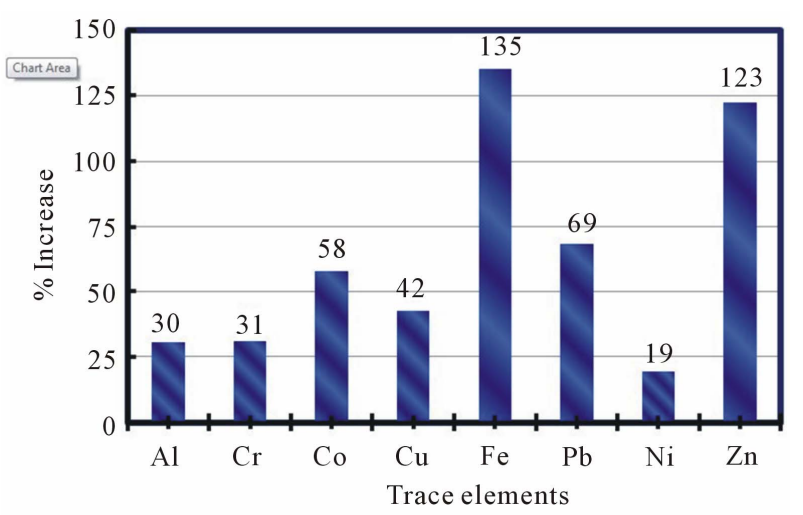

Figure 2. Variations in the increases of various trace elements in indoor household drinking water (I-HW) for all of the samples collected from the 99 sampling locations. 
Table 2. Mean values of the concentrations of trace elements in the indoor (I-HW) and outdoor (O-HW) household drinking water at various locations in different governorates ${ }^{\mathrm{a}}$.

(a)

\begin{tabular}{|c|c|c|c|c|c|c|}
\hline \multirow{2}{*}{ Element-I/O ${ }^{\mathbf{b}}$} & \multicolumn{6}{|c|}{ Sampling locations } \\
\hline & CKF & CQB & CSE & CSH & CSL & CSO \\
\hline Al-I & $34.4 \pm 10.7$ & $13.4 \pm 18.2$ & $3.17 \pm 2.39$ & $9.94 \pm 3.59$ & $5.55 \pm 4.89$ & $22.4 \pm 21.6$ \\
\hline Al-O & $33.5 \pm 11.7$ & $4.50 \pm 3.21$ & $1.69 \pm 0.99$ & $5.60 \pm 2.39$ & $4.07 \pm 3.17$ & $10.6 \pm 2.00$ \\
\hline As-I & $0.43 \pm 0.07$ & $0.12 \pm 0.06$ & $0.32 \pm 0.09$ & $0.36 \pm 0.10$ & $0.45 \pm 0.06$ & $0.13 \pm 0.05$ \\
\hline As-O & $0.43 \pm 0.07$ & $0.24 \pm 0.15$ & $0.32 \pm 0.13$ & $0.22 \pm 0.05$ & $0.47 \pm 0.08$ & $0.11 \pm 0.03$ \\
\hline B-I & $127 \pm 15.2$ & $164 \pm 39.5$ & $120 \pm 11.2$ & $211 \pm 55.7$ & $128 \pm 16.7$ & $67.8 \pm 11.6$ \\
\hline B-O & $128 \pm 15.3$ & $153 \pm 30.4$ & $120 \pm 12.4$ & $209 \pm 184$ & $130 \pm 13.5$ & $65.6 \pm 12.1$ \\
\hline Ba-I & $3.39 \pm 0.36$ & $6.99 \pm 9.75$ & $2.29 \pm 0.30$ & $2.49 \pm 0.33$ & $2.57 \pm 0.40$ & $4.41 \pm 0.48$ \\
\hline Ba-O & $3.37 \pm 0.36$ & $2.54 \pm 0.29$ & $2.27 \pm 0.03$ & $2.43 \pm 0.35$ & $2.55 \pm 0.37$ & $4.14 \pm 0.48$ \\
\hline Ba-I & $3.39 \pm 0.36$ & $6.99 \pm 9.75$ & $2.29 \pm 0.30$ & $2.49 \pm 0.33$ & $2.57 \pm 0.40$ & $4.41 \pm 0.48$ \\
\hline Ba-O & $3.37 \pm 0.36$ & $2.54 \pm 0.29$ & $2.27 \pm 0.03$ & $2.43 \pm 0.35$ & $2.55 \pm 0.37$ & $4.14 \pm 0.48$ \\
\hline Ca-I & $53.0 \pm 4.31$ & $43.1 \pm 4.43$ & $51.0 \pm 3.01$ & $47.4 \pm 5.70$ & $51.7 \pm 4.32$ & $32.4 \pm 3.71$ \\
\hline Ca-O & $52.4 \pm 4.93$ & $43.2 \pm 6.42$ & $51.2 \pm 3.33$ & $47.5 \pm 6.10$ & $52.2 \pm 3.94$ & $29.9 \pm 7.42$ \\
\hline Cd-I & $0.11 \pm 0.00$ & $0.07 \pm 0.00$ & $0.10 \pm 0.00$ & $0.16 \pm 0.00$ & $0.07 \pm 0.00$ & $0.13 \pm 0.04$ \\
\hline Cd-O & $0.11 \pm 0.00$ & $0.08 \pm 0.00$ & $0.10 \pm 0.01$ & $1.58 \pm 0.00$ & $0.07 \pm 0.00$ & $0.11 \pm 0.00$ \\
\hline Co-I & $0.59 \pm 0.66$ & $0.51 \pm 0.70$ & $0.39 \pm 0.50$ & $8.14 \pm 14.0$ & $4.06 \pm 8.52$ & $0.16 \pm 0.16$ \\
\hline Co-O & $0.58 \pm 0.63$ & $0.30 \pm 0.60$ & $0.36 \pm 0.47$ & $0.60 \pm 0.92$ & $0.57 \pm 0.79$ & $0.10 \pm 0.08$ \\
\hline Cr-I & $0.63 \pm 0.31$ & $0.23 \pm 0.08$ & $0.60 \pm 0.16$ & $0.61 \pm 0.18$ & $1.33 \pm 1.72$ & $0.21 \pm 0.03$ \\
\hline Cr-O & $0.61 \pm 0.30$ & $0.17 \pm 0.09$ & $0.55 \pm 0.18$ & $0.53 \pm 0.25$ & $0.55 \pm 0.30$ & $0.17 \pm 0.04$ \\
\hline Cu-I & $31.8 \pm 6.72$ & $54.4 \pm 57.0$ & $50.0 \pm 23.4$ & $52.7 \pm 16.4$ & $59.6 \pm 7.5$ & $60.8 \pm 29.2$ \\
\hline $\mathrm{Cu}-\mathrm{O}$ & $31.4 \pm 6.79$ & $26.7 \pm 16.2$ & $41.8 \pm 12.4$ & $17.3 \pm 6.78$ & $48.5 \pm 13.0$ & $28.5 \pm 7.86$ \\
\hline Fe-I & $9.54 \pm 2.51$ & $576 \pm 534$ & $92.4 \pm 104$ & $98.0 \pm 22.8$ & $15.5 \pm 8.6$ & $167 \pm 128$ \\
\hline Fe-O & $8.48 \pm 2.67$ & $340 \pm 336$ & $22.6 \pm 23.1$ & $16.4 \pm 4.47$ & $8.73 \pm 1.65$ & $22.6 \pm 20.6$ \\
\hline Pb-I & $0.55 \pm 0.50$ & $0.61 \pm 1.08$ & $0.65 \pm 0.34$ & $6.26 \pm 10.7$ & $0.40 \pm 0.45$ & $0.17 \pm 0.14$ \\
\hline Pb-O & $0.49 \pm 0.43$ & $0.51 \pm 0.94$ & $0.26 \pm 0.27$ & $0.50 \pm 0.63$ & $0.19 \pm 0.29$ & $0.19 \pm 0.15$ \\
\hline Mg-I & $13.5 \pm 1.82$ & $12.9 \pm 0.92$ & $12.7 \pm 1.41$ & $13.2 \pm 1.52$ & $13.5 \pm 1.82$ & $6.08 \pm 1.44$ \\
\hline Mg-O & $13.5 \pm 1.94$ & $12.7 \pm 1.34$ & $12.7 \pm 1.43$ & $13.2 \pm 1.61$ & $13.7 \pm 1.64$ & $6.08 \pm 1.68$ \\
\hline Mn-I & $0.99 \pm 0.47$ & $9.46 \pm 2.96$ & $0.82 \pm 0.87$ & $2.15 \pm 2.98$ & $1.13 \pm 0.41$ & $1.85 \pm 1.06$ \\
\hline Mn-O & $0.92 \pm 0.38$ & $10.2 \pm 6.13$ & $1.14 \pm 2.05$ & $2.71 \pm 2.04$ & $3.66 \pm 6.48$ & $1.39 \pm 1.25$ \\
\hline Hg-I & $0.76 \pm 0.00$ & $0.42 \pm 0.00$ & $0.80 \pm 0.00$ & $0.53 \pm 0.00$ & $0.90 \pm 0.00$ & $0.12 \pm 0.00$ \\
\hline Hg-O & $0.62 \pm 0.00$ & $0.32 \pm 0.00$ & $0.80 \pm 0.00$ & $0.79 \pm 0.00$ & $0.40 \pm 0.00$ & $0.08 \pm 0.04$ \\
\hline Mo-I & $8.97 \pm 2.92$ & $5.38 \pm 2.14$ & $8.31 \pm 2.50$ & $8.21 \pm 2.53$ & $9.37 \pm 3.51$ & $3.32 \pm 1.17$ \\
\hline Mo-O & $8.73 \pm 3.23$ & $5.57 \pm 2.76$ & $8.65 \pm 2.01$ & $14.7 \pm 15.1$ & $15.5 \pm 16.6$ & $3.22 \pm 1.02$ \\
\hline Ni-I & $5.98 \pm 1.43$ & $10.2 \pm 3.04$ & $7.63 \pm 1.52$ & $9.94 \pm 10.6$ & $9.29 \pm 7.89$ & $3.65 \pm 1.34$ \\
\hline Ni-O & $5.57 \pm 1.13$ & $7.90 \pm 1.55$ & $7.38 \pm 1.73$ & $5.03 \pm 0.82$ & $5.80 \pm 0.61$ & $3.02 \pm 1.17$ \\
\hline K-I & $1.54 \pm 0.19$ & $1.53 \pm 0.13$ & $1.50 \pm 0.16$ & $1.68 \pm 0.20$ & $1.55 \pm 0.19$ & $1.74 \pm 0.25$ \\
\hline K-O & $1.55 \pm 0.20$ & $1.52 \pm 0.13$ & $1.49 \pm 0.17$ & $1.66 \pm 0.26$ & $1.57 \pm 0.19$ & $1.73 \pm 0.26$ \\
\hline Sb-I & $1.04 \pm 0.00$ & $0.25 \pm 0.15$ & $0.98 \pm 0.53$ & $2.15 \pm 0.00$ & $1.71 \pm 0.37$ & $1.70 \pm 0.00$ \\
\hline Sb-O & $0.39 \pm 0.50$ & $0.50 \pm 0.40$ & $0.57 \pm 0.45$ & $2.79 \pm 0.00$ & $2.66 \pm 0.08$ & $0.93 \pm 0.39$ \\
\hline Se-I & $0.95 \pm 0.15$ & $0.53 \pm 0.21$ & $0.79 \pm 0.14$ & $0.81 \pm 0.05$ & $0.81 \pm 0.27$ & $0.22 \pm 0.04$ \\
\hline Se-O & $1.03 \pm 0.29$ & $0.55 \pm 0.29$ & $0.79 \pm 0.14$ & $0.81 \pm 0.15$ & $0.93 \pm 0.17$ & $0.22 \pm 0.05$ \\
\hline Na-I & $52.9 \pm 7.10$ & $52.3 \pm 3.86$ & $52.0 \pm 4.42$ & $56.7 \pm 6.71$ & $53.0 \pm 6.70$ & $36.7 \pm 4.82$ \\
\hline Na-O & $52.8 \pm 7.90$ & $51.0 \pm 5.04$ & $51.8 \pm 4.54$ & $57.4 \pm 10.1$ & $53.7 \pm 6.54$ & $36.6 \pm 5.53$ \\
\hline Sr-I & $1.27 \pm 0.15$ & $1.19 \pm 0.08$ & $1.18 \pm 0.12$ & $1.27 \pm 0.13$ & $1.28 \pm 0.17$ & $0.60 \pm 0.16$ \\
\hline Sr-O & $1.27 \pm 0.14$ & $1.16 \pm 0.14$ & $1.18 \pm 0.12$ & $1.23 \pm 0.11$ & $1.30 \pm 0.16$ & $0.59 \pm 0.17$ \\
\hline Ti-I & $0.20 \pm 0.05$ & $0.16 \pm 0.04$ & $0.13 \pm 0.04$ & $0.23 \pm 0.07$ & $0.25 \pm 0.14$ & $0.23 \pm 0.06$ \\
\hline Ti-O & $0.19 \pm 0.05$ & $0.14 \pm 0.03$ & $0.14 \pm 0.04$ & $0.15 \pm 0.05$ & $0.20 \pm 0.09$ & $0.24 \pm 0.12$ \\
\hline V-I & $2.87 \pm 0.23$ & $0.93 \pm 0.43$ & $2.15 \pm 0.42$ & $2.58 \pm 0.11$ & $2.71 \pm 0.24$ & $0.78 \pm 0.08$ \\
\hline V-O & $2.86 \pm 0.21$ & $1.63 \pm 0.93$ & $2.15 \pm 0.73$ & $1.53 \pm 0.22$ & $2.81 \pm 0.36$ & $0.82 \pm 0.01$ \\
\hline Zn-I & $16.3 \pm 4.13$ & $231 \pm 140$ & $74.6 \pm 91.3$ & $194 \pm 105$ & $52.3 \pm 14.3$ & $78.8 \pm 42.8$ \\
\hline Zn-O & $8.70 \pm 0.55$ & $154 \pm 105$ & $27.7 \pm 12.3$ & $27.7 \pm 4.63$ & $37.1 \pm 14.0$ & $15.9 \pm 4.88$ \\
\hline No. samples ${ }^{c}$ & 7 & 7 & 19 & 7 & 7 & 6 \\
\hline
\end{tabular}


(b)

\begin{tabular}{|c|c|c|c|c|c|c|}
\hline \multirow{2}{*}{ Element-I/O $\mathbf{O}^{\mathbf{b}}$} & \multicolumn{6}{|c|}{ Sampling locations, contd. } \\
\hline & CSW & DUH & FAR & FRI & HSL & JOY \\
\hline Al-I & $6.19 \pm 3.50$ & $10.4 \pm 4.83$ & $6.25 \pm 10.8$ & $8.98 \pm 4.03$ & $5.55 \pm 2.38$ & $6.76 \pm 3.86$ \\
\hline Al-O & $3.79 \pm 3.12$ & $8.16 \pm 3.04$ & $5.09 \pm 8.56$ & $8.35 \pm 4.15$ & $5.23 \pm 2.30$ & $5.96 \pm 3.50$ \\
\hline As-I & $0.26 \pm 0.10$ & $0.05 \pm 0.01$ & $0.47 \pm 0.08$ & $0.41 \pm 0.08$ & $0.28 \pm 0.07$ & $0.38 \pm 0.09$ \\
\hline As-O & $0.30 \pm 0.13$ & $0.07 \pm 0.03$ & $0.52 \pm 0.10$ & $0.41 \pm 0.08$ & $0.28 \pm 0.07$ & $0.39 \pm 0.09$ \\
\hline B-I & $110 \pm 13.0$ & $45.8 \pm 7.7$ & $127 \pm 11.6$ & $123 \pm 13.4$ & $94.6 \pm 16.4$ & $132 \pm 11.3$ \\
\hline B-O & $111 \pm 20.5$ & $45.9 \pm 7.4$ & $129 \pm 16.0$ & $124 \pm 15.3$ & $94.1 \pm 15.9$ & $130 \pm 9.6$ \\
\hline Ba-I & $2.37 \pm 0.31$ & $1.50 \pm 0.22$ & $2.29 \pm 0.35$ & $2.20 \pm 0.33$ & $5.10 \pm 0.65$ & $2.89 \pm 0.22$ \\
\hline Ba-O & $2.27 \pm 0.30$ & $1.55 \pm 0.29$ & $2.19 \pm 0.30$ & $2.21 \pm 0.42$ & $5.10 \pm 0.65$ & $2.88 \pm 0.19$ \\
\hline Ba-I & $2.37 \pm 0.31$ & $1.50 \pm 0.22$ & $2.29 \pm 0.35$ & $2.20 \pm 0.33$ & $5.10 \pm 0.65$ & $2.89 \pm 0.22$ \\
\hline Ba-O & $2.27 \pm 0.30$ & $1.55 \pm 0.29$ & $2.19 \pm 0.30$ & $2.21 \pm 0.42$ & $5.10 \pm 0.65$ & $2.88 \pm 0.19$ \\
\hline Ca-I & $45.5 \pm 3.9$ & $26.1 \pm 5.1$ & $52.0 \pm 3.9$ & $51.8 \pm 3.3$ & $38.3 \pm 5.0$ & $49.4 \pm 4.0$ \\
\hline Ca-O & $44.2 \pm 10.2$ & $26.4 \pm 5.0$ & $51.9 \pm 4.5$ & $53.0 \pm 4.3$ & $37.9 \pm 5.2$ & $49.4 \pm 3.6$ \\
\hline Cd-I & $0.24 \pm 0.00$ & $0.08 \pm 0.00$ & $0.18 \pm 0.00$ & $0.10 \pm 0.02$ & $0.07 \pm 0.00$ & $0.07 \pm 0.00$ \\
\hline Cd-O & $0.16 \pm 0.00$ & $0.07 \pm 0.00$ & $0.15 \pm 0.00$ & $0.11 \pm 0.04$ & $0.07 \pm 0.00$ & $0.10 \pm 0.00$ \\
\hline Co-I & $0.41 \pm 0.65$ & $0.64 \pm 0.74$ & $0.28 \pm 0.27$ & $0.34 \pm 0.52$ & $0.48 \pm 0.51$ & $0.39 \pm 0.64$ \\
\hline Co-O & $0.31 \pm 0.49$ & $0.21 \pm 0.20$ & $0.29 \pm 0.29$ & $0.40 \pm 0.49$ & $0.45 \pm 0.39$ & $0.32 \pm 0.62$ \\
\hline Cr-I & $0.73 \pm 0.25$ & $0.13 \pm 0.13$ & $0.86 \pm 0.52$ & $0.66 \pm 0.16$ & $0.60 \pm 10$ & $1.47 \pm 0.69$ \\
\hline Cr-O & $0.41 \pm 0.24$ & $0.09 \pm 0.03$ & $0.61 \pm 0.18$ & $0.61 \pm 0.15$ & $0.58 \pm 0.10$ & $0.92 \pm 0.26$ \\
\hline Cu-I & $71.4 \pm 15.3$ & $51.9 \pm 13.8$ & $129 \pm 45.6$ & $58.5 \pm 9.2$ & $46.1 \pm 10.4$ & $35.4 \pm 7.2$ \\
\hline $\mathrm{Cu}-\mathrm{O}$ & $55.2 \pm 16.3$ & $33.0 \pm 9.27$ & $50.4 \pm 9.66$ & $46.3 \pm 8.1$ & $44.3 \pm 10.1$ & $33.3 \pm 6.64$ \\
\hline Fe-I & $50.9 \pm 9.74$ & $16.7 \pm 13.7$ & $18.4 \pm 6.10$ & $26.7 \pm 9.6$ & $31.1 \pm 38.4$ & $20.4 \pm 11.2$ \\
\hline Fe-O & $10.7 \pm 6.81$ & $8.18 \pm 2.09$ & $7.36 \pm 2.74$ & $15.1 \pm 6.4$ & $29.7 \pm 37.3$ & $16.6 \pm 7.19$ \\
\hline Pb-I & $1.03 \pm 1.43$ & $0.85 \pm 1.30$ & $0.32 \pm 0.45$ & $0.36 \pm 0.38$ & $0.23 \pm 0.28$ & $0.33 \pm 0.45$ \\
\hline Pb-O & $0.63 \pm 0.70$ & $0.18 \pm 0.14$ & $0.27 \pm 0.42$ & $0.24 \pm 0.32$ & $0.18 \pm 0.24$ & $0.24 \pm 0.37$ \\
\hline Mg-I & $13.2 \pm 0.9$ & $3.68 \pm 0.85$ & $13.5 \pm 1.9$ & $12.1 \pm 3.4$ & $9.44 \pm 1.81$ & $13.8 \pm 1.3$ \\
\hline Mg-O & $12.4 \pm 3.5$ & $3.73 \pm 0.84$ & $13.7 \pm 2.0$ & $12.2 \pm 3.5$ & $9.43 \pm 1.76$ & $13.6 \pm 1.24$ \\
\hline Mn-I & $1.70 \pm 0.8$ & $0.90 \pm 0.22$ & $1.08 \pm 0.33$ & $1.53 \pm 0.47$ & $1.72 \pm 0.99$ & $1.51 \pm 0.88$ \\
\hline Mn-O & $1.31 \pm 0.87$ & $1.61 \pm 1.45$ & $0.90 \pm 0.39$ & $1.33 \pm 0.52$ & $1.71 \pm 1.03$ & $1.12 \pm 0.43$ \\
\hline Hg-I & $0.12 \pm 0.00$ & ND & $0.15 \pm 0.00$ & $0.11 \pm 0.00$ & $0.18 \pm 0.00$ & ND \\
\hline Hg-O & $0.10 \pm 0.02$ & ND & $0.13 \pm 0.00$ & $0.11 \pm 0.00$ & $0.13 \pm 0.02$ & ND \\
\hline Mo-I & $6.32 \pm 1.98$ & $2.42 \pm 0.73$ & $7.86 \pm 4.53$ & $8.49 \pm 1.98$ & $6.19 \pm 1.65$ & $11.04 \pm 1.98$ \\
\hline Mo-O & $6.01 \pm 3.12$ & $2.46 \pm 0.76$ & $8.24 \pm 4.74$ & $8.75 \pm 2.02$ & $6.18 \pm 1.72$ & $10.89 \pm 1.54$ \\
\hline Ni-I & $12.8 \pm 4.09$ & $2.09 \pm 0.55$ & $7.44 \pm 3.24$ & $6.29 \pm 1.62$ & $7.04 \pm 1.25$ & $7.45 \pm 1.51$ \\
\hline $\mathrm{Ni}-\mathrm{O}$ & $9.34 \pm 3.55$ & $1.80 \pm 0.47$ & $6.63 \pm 2.78$ & $5.97 \pm 1.55$ & $6.88 \pm 1.27$ & $6.54 \pm 1.46$ \\
\hline K-I & $1.77 \pm 0.16$ & $1.43 \pm 0.35$ & $1.57 \pm 0.21$ & $1.49 \pm 0.15$ & $1.50 \pm 0.23$ & $1.72 \pm 0.16$ \\
\hline K-O & $1.48 \pm 0.21$ & $1.46 \pm 0.35$ & $1.57 \pm 0.23$ & $1.50 \pm 0.18$ & $1.50 \pm 0.22$ & $1.71 \pm 0.13$ \\
\hline Sb-I & $0.55 \pm 0.45$ & $0.61 \pm 0.34$ & $0.80 \pm 0.98$ & $0.47 \pm 0.31$ & $0.34 \pm 0.08$ & $1.11 \pm 0.81$ \\
\hline Sb-O & $0.61 \pm 0.19$ & $0.70 \pm 0.46$ & $0.97 \pm 1.29$ & $0.34 \pm 0.37$ & $0.45 \pm 0.20$ & $1.49 \pm 1.08$ \\
\hline Se-I & $0.72 \pm 0.59$ & $0.16 \pm 0.11$ & $0.91 \pm 0.12$ & $0.82 \pm 0.18$ & $0.55 \pm 0.19$ & $0.89 \pm 0.16$ \\
\hline Se-O & $0.59 \pm 0.38$ & $0.16 \pm 0.10$ & $0.94 \pm 0.16$ & $0.91 \pm 0.33$ & $0.65 \pm 0.36$ & $0.90 \pm 0.14$ \\
\hline Na-I & $54.4 \pm 3.8$ & $28.6 \pm 5.5$ & $53.4 \pm 5.6$ & $53.5 \pm 5.6$ & $44.4 \pm 5.3$ & $56.6 \pm 3.9$ \\
\hline Na-O & $50.9 \pm 10.3$ & $28.9 \pm 5.2$ & $53.1 \pm 7.8$ & $54.1 \pm 6.2$ & $43.9 \pm 5.6$ & $56.6 \pm 3.7$ \\
\hline Sr-I & $1.16 \pm 0.09$ & $0.37 \pm 0.10$ & $1.28 \pm 0.11$ & $1.20 \pm 0.13$ & $0.90 \pm 0.17$ & $1.29 \pm 0.14$ \\
\hline Sr-O & $1.18 \pm 0.13$ & $0.38 \pm 0.10$ & $1.30 \pm 0.15$ & $1.22 \pm 0.16$ & $0.90 \pm 0.17$ & $1.28 \pm 0.13$ \\
\hline Ti-I & $0.14 \pm 0.04$ & $0.14 \pm 0.08$ & $0.23 \pm 0.06$ & $0.16 \pm 0.06$ & $0.21 \pm 0.09$ & $0.19 \pm 0.09$ \\
\hline Ti-O & $0.13 \pm 0.04$ & $0.51 \pm 0.55$ & $0.18 \pm 0.04$ & $0.19 \pm 0.07$ & $0.21 \pm 0.10$ & $0.16 \pm 0.05$ \\
\hline V-I & $1.62 \pm 0.53$ & $0.50 \pm 0.13$ & $2.95 \pm 0.21$ & $2.64 \pm 0.33$ & $1.85 \pm 0.35$ & $2.44 \pm 0.48$ \\
\hline V-O & $1.84 \pm 0.82$ & $0.58 \pm 0.15$ & $3.10 \pm 0.24$ & $2.70 \pm 0.33$ & $1.86 \pm 0.35$ & $2.54 \pm 0.43$ \\
\hline Zn-I & $27.6 \pm 7.13$ & $31.7 \pm 8.87$ & $67.5 \pm 31.2$ & $40.7 \pm 11.6$ & $28.7 \pm 6.4$ & $31.5 \pm 17.0$ \\
\hline Zn-O & $22.0 \pm 6.16$ & $19.4 \pm 4.80$ & $19.8 \pm 5.25$ & $34.6 \pm 6.90$ & $22.6 \pm 6.87$ & $11.0 \pm 2.90$ \\
\hline No. samples ${ }^{c}$ & 19 & 19 & 7 & 20 & 19 & 19 \\
\hline
\end{tabular}


(c)

\begin{tabular}{|c|c|c|c|c|c|c|c|}
\hline \multirow{2}{*}{ Element-I/O ${ }^{\mathbf{b}}$} & \multicolumn{7}{|c|}{ Governorates $^{\mathrm{d}}$} \\
\hline & GAH & GCA & GFA & GHA & GJA & GMK & All $^{\mathrm{e}}$ \\
\hline Al-I & $14.6 \pm 7.63$ & $12.4 \pm 13.1$ & $8.35 \pm 5.96$ & $8.09 \pm 4.95$ & $7.74 \pm 4.64$ & $20.5 \pm 6.61$ & $11.7 \pm 10.1$ \\
\hline Al-O & $11.1 \pm 5.47$ & $8.51 \pm 9.04$ & $7.14 \pm 4.95$ & $7.28 \pm 4.68$ & $6.13 \pm 3.71$ & $18.8 \pm 6.97$ & $8.71 \pm 7.37$ \\
\hline As-I & $0.08 \pm 0.07$ & $0.28 \pm 0.13$ & $0.43 \pm 0.08$ & $0.28 \pm 0.07$ & $0.35 \pm 0.10$ & $0.06 \pm 0.00$ & $0.29 \pm 0.14$ \\
\hline As-O & $0.07 \pm 0.03$ & $0.29 \pm 0.14$ & $0.44 \pm 0.10$ & $0.28 \pm 0.07$ & $0.39 \pm 0.07$ & $0.14 \pm 0.13$ & $0.30 \pm 0.15$ \\
\hline B-I & $46.2 \pm 7.3$ & $129 \pm 43.7$ & $125 \pm 12.5$ & $94.6 \pm 16.4$ & $125 \pm 16.5$ & NA & $116 \pm 40.0$ \\
\hline B-O & $45.9 \pm 7.0$ & $128 \pm 68.0$ & $126 \pm 15.1$ & $94.1 \pm 15.9$ & $121 \pm 15.3$ & NA & $115 \pm 53.6$ \\
\hline Ba-I & $1.91 \pm 1.26$ & $3.19 \pm 3.31$ & $2.23 \pm 0.33$ & $5.10 \pm 0.65$ & $2.68 \pm 0.49$ & NA & $3.03 \pm 2.45$ \\
\hline Ba-O & $1.88 \pm 1.07$ & $2.63 \pm 0.67$ & $2.20 \pm 0.37$ & $5.10 \pm 0.65$ & $2.63 \pm 0.48$ & NA & $2.74 \pm 1.06$ \\
\hline Ca-I & $27.1 \pm 3.9$ & $46.4 \pm 6.9$ & $51.9 \pm 3.4$ & $38.3 \pm 5.0$ & $47.3 \pm 4.2$ & $28.4 \pm 0.3$ & $43.3 \pm 9.6$ \\
\hline Ca-O & $27.3 \pm 3.9$ & $45.6 \pm 8.9$ & $52.6 \pm 4.3$ & $37.9 \pm 5.2$ & $47.3 \pm 4.1$ & $28.5 \pm 0.5$ & $42.9 \pm 10.4$ \\
\hline Cd-I & $0.07 \pm 0.01$ & $0.12 \pm 0.06$ & $0.13 \pm 0.04$ & $0.07 \pm 0.01$ & $0.12 \pm 0.04$ & $0.07 \pm 0.01$ & $0.11 \pm 0.05$ \\
\hline Cd-O & $0.07 \pm 0.01$ & $0.15 \pm 0.25$ & $0.12 \pm 0.04$ & $0.08 \pm 0.01$ & $0.13 \pm 0.05$ & $0.07 \pm 0.01$ & $0.12 \pm 0.17$ \\
\hline Co-I & $0.53 \pm 0.72$ & $1.20 \pm 3.34$ & $0.66 \pm 0.52$ & $0.67 \pm 0.53$ & $0.59 \pm 0.64$ & $0.47 \pm 0.32$ & $0.82 \pm 2.10$ \\
\hline Co-O & $0.26 \pm 0.22$ & $0.59 \pm 0.57$ & $0.61 \pm 0.47$ & $0.54 \pm 0.45$ & $0.51 \pm 0.59$ & $0.40 \pm 0.27$ & $0.52 \pm 0.50$ \\
\hline Cr-I & $0.37 \pm 0.32$ & $0.63 \pm 0.53$ & $0.77 \pm 0.30$ & $0.55 \pm 0.20$ & $1.12 \pm 0.58$ & $0.43 \pm 0.23$ & $0.68 \pm 0.49$ \\
\hline Cr-O & $0.25 \pm 0.21$ & $0.48 \pm 0.29$ & $0.66 \pm 0.26$ & $0.49 \pm 0.21$ & $0.79 \pm 0.28$ & $0.29 \pm 0.13$ & $0.52 \pm 0.31$ \\
\hline Cu-I & $44.7 \pm 39.5$ & $54.1 \pm 26.2$ & $69.8 \pm 39.3$ & $41.2 \pm 12.5$ & $43.0 \pm 10.9$ & $39.7 \pm 25.7$ & $51.9 \pm 30.1$ \\
\hline $\mathrm{Cu}-\mathrm{O}$ & $27.6 \pm 18.9$ & $38.3 \pm 15.8$ & $43.8 \pm 10.5$ & $35.8 \pm 14.1$ & $35.1 \pm 10.3$ & $26.1 \pm 11.8$ & $36.4 \pm 15.4$ \\
\hline Fe-I & $64.5 \pm 215$ & $103 \pm 209$ & $35.0 \pm 50.0$ & $31.6 \pm 31.1$ & $42.1 \pm 36.7$ & $19.3 \pm 9.72$ & $68.4 \pm 164$ \\
\hline Fe-O & $14.5 \pm 10.6$ & $42.6 \pm 124$ & $15.9 \pm 8.88$ & $27.2 \pm 30.5$ & $26.4 \pm 29.9$ & $11.6 \pm 9.96$ & $29.1 \pm 82.3$ \\
\hline Pb-I & $0.58 \pm 0.76$ & $1.12 \pm 2.30$ & $0.87 \pm 0.53$ & $0.49 \pm 0.32$ & $0.76 \pm 0.68$ & $0.92 \pm 1.21$ & $0.86 \pm 1.52$ \\
\hline Pb-O & $0.32 \pm 0.19$ & $0.57 \pm 0.49$ & $0.68 \pm 0.51$ & $0.36 \pm 0.28$ & $0.58 \pm 0.56$ & $0.37 \pm 0.19$ & $0.51 \pm 0.45$ \\
\hline Mg-I & $3.80 \pm 0.96$ & $12.4 \pm 2.50$ & $12.5 \pm 3.0$ & $9.44 \pm 1.81$ & $12.9 \pm 1.6$ & $3.45 \pm 0.34$ & $10.8 \pm 3.9$ \\
\hline Mg-O & $3.71 \pm 0.87$ & $12.2 \pm 2.90$ & $12.6 \pm 3.1$ & $9.43 \pm 1.76$ & $12.9 \pm 1.5$ & $3.42 \pm 0.34$ & $10.7 \pm 4.0$ \\
\hline Mn-I & $1.66 \pm 2.83$ & $2.30 \pm 2.77$ & $1.84 \pm 2.08$ & $1.55 \pm 0.86$ & $1.65 \pm 0.92$ & $1.48 \pm 1.59$ & $1.93 \pm 2.30$ \\
\hline Mn-O & $1.97 \pm 3.18$ & $2.45 \pm 3.32$ & $1.71 \pm 1.63$ & $1.58 \pm 0.87$ & $1.71 \pm 1.01$ & $1.42 \pm 0.89$ & $2.02 \pm 2.59$ \\
\hline Hg-I & $0.63 \pm 0.46$ & $0.36 \pm 0.34$ & $0.29 \pm 0.29$ & $0.39 \pm 0.33$ & $0.84 \pm 1.10$ & $0.24 \pm 0.26$ & $0.42 \pm 0.45$ \\
\hline Hg-O & $0.62 \pm 0.48$ & $0.36 \pm 0.34$ & $0.25 \pm 0.23$ & $0.40 \pm 0.39$ & $1.06 \pm 1.07$ & $0.25 \pm 0.20$ & $0.43 \pm 0.46$ \\
\hline Mo-I & $2.59 \pm 0.84$ & $6.24 \pm 2.92$ & $7.31 \pm 2.79$ & $5.27 \pm 2.50$ & $8.44 \pm 3.63$ & $2.99 \pm 1.54$ & $5.98 \pm 3.23$ \\
\hline Mo-O & $2.56 \pm 0.93$ & $7.04 \pm 6.56$ & $7.63 \pm 2.80$ & $5.21 \pm 2.44$ & $8.46 \pm 3.37$ & $3.02 \pm 1.55$ & $6.33 \pm 4.96$ \\
\hline Ni-I & $2.95 \pm 1.90$ & $8.33 \pm 4.75$ & $6.63 \pm 1.83$ & $5.61 \pm 2.02$ & $6.72 \pm 1.48$ & $3.16 \pm 2.25$ & $6.56 \pm 3.83$ \\
\hline Ni-O & $2.32 \pm 1.26$ & $6.67 \pm 2.91$ & $5.90 \pm 1.75$ & $5.25 \pm 2.10$ & $5.95 \pm 1.59$ & $2.56 \pm 2.12$ & $5.50 \pm 2.74$ \\
\hline K-I & $1.36 \pm 0.26$ & $1.62 \pm 0.19$ & $1.51 \pm 0.17$ & $1.50 \pm 0.23$ & $1.61 \pm 0.23$ & $1.30 \pm 0.02$ & $1.55 \pm 0.23$ \\
\hline K-O & $1.38 \pm 0.27$ & $1.55 \pm 0.20$ & $1.52 \pm 0.19$ & $1.50 \pm 0.22$ & $1.61 \pm 0.22$ & $1.30 \pm 0.02$ & $1.52 \pm 0.22$ \\
\hline Sb-I & $0.78 \pm 0.42$ & $1.30 \pm 0.92$ & $0.69 \pm 0.64$ & $0.54 \pm 0.51$ & $1.35 \pm 1.11$ & $1.06 \pm 0.59$ & $0.98 \pm 0.79$ \\
\hline Sb-O & $0.91 \pm 0.46$ & $1.24 \pm 1.17$ & $0.70 \pm 0.80$ & $0.55 \pm 0.44$ & $1.50 \pm 0.98$ & $0.97 \pm 0.57$ & $1.00 \pm 0.91$ \\
\hline Se-I & $0.44 \pm 0.38$ & $0.82 \pm 0.48$ & $1.05 \pm 1.40$ & $1.08 \pm 1.21$ & $0.78 \pm 0.38$ & $1.08 \pm 0.95$ & $0.86 \pm 0.84$ \\
\hline Se-O & $0.35 \pm 0.31$ & $0.79 \pm 0.39$ & $1.08 \pm 0.91$ & $1.28 \pm 1.45$ & $0.86 \pm 0.49$ & $1.72 \pm 0.88$ & $0.89 \pm 0.77$ \\
\hline Na-I & $28.5 \pm 5.1$ & $52.0 \pm 7.6$ & $50.4 \pm 13.8$ & $44.4 \pm 5.3$ & $53.6 \pm 6.1$ & $25.9 \pm 1.2$ & $47.3 \pm 12.0$ \\
\hline Na-O & $28.6 \pm 5.0$ & $51.3 \pm 9.0$ & $50.7 \pm 14.3$ & $43.9 \pm 5.6$ & $53.4 \pm 6.2$ & $26.2 \pm 1.2$ & $46.8 \pm 12.4$ \\
\hline Sr-I & $0.36 \pm 0.09$ & $1.15 \pm 0.22$ & $1.23 \pm 0.13$ & $0.90 \pm 0.17$ & $1.20 \pm 0.18$ & NA & $1.07 \pm 0.31$ \\
\hline Sr-O & $0.37 \pm 0.09$ & $1.15 \pm 0.23$ & $1.25 \pm 0.16$ & $0.90 \pm 0.17$ & $1.19 \pm 0.17$ & NA & $1.07 \pm 0.32$ \\
\hline Ti-I & $0.16 \pm 0.10$ & $0.18 \pm 0.08$ & $0.19 \pm 0.06$ & $0.21 \pm 0.09$ & $0.16 \pm 0.08$ & NA & $0.18 \pm 0.08$ \\
\hline Ti-O & $0.48 \pm 0.51$ & $0.16 \pm 0.06$ & $0.19 \pm 0.06$ & $0.21 \pm 0.10$ & $0.15 \pm 0.06$ & NA & $0.19 \pm 0.17$ \\
\hline V-I & $0.49 \pm 0.12$ & $1.94 \pm 0.79$ & $2.75 \pm 0.32$ & $1.85 \pm 0.35$ & $2.25 \pm 0.58$ & NA & $1.97 \pm 0.83$ \\
\hline V-O & $0.57 \pm 0.14$ & $1.98 \pm 0.83$ & $2.83 \pm 0.35$ & $1.86 \pm 0.35$ & $2.46 \pm 0.37$ & NA & $2.04 \pm 0.89$ \\
\hline Zn-I & $44.1 \pm 55.2$ & $70.5 \pm 91.0$ & $48.9 \pm 42.9$ & $30.7 \pm 17.4$ & $42.4 \pm 59.5$ & $26.1 \pm 11.5$ & $53.5 \pm 69.7$ \\
\hline Zn-O & $18.6 \pm 16.0$ & $32.0 \pm 48.7$ & $22.2 \pm 11.4$ & $23.3 \pm 19.8$ & $13.3 \pm 5.96$ & $13.3 \pm 7.76$ & $24.1 \pm 33.2$ \\
\hline No. samples ${ }^{c}$ & 46 & 128 & 56 & 37 & 42 & 12 & 321 \\
\hline No. locations & 16 & 38 & 19 & 11 & 9 & 6 & 99 \\
\hline
\end{tabular}

a Concentrations of elements are in $\mu \mathrm{g} \cdot \mathrm{L}^{-1}$, except for $\mathrm{Ca}, \mathrm{Mg}, \mathrm{Na}$, and $\mathrm{Sr}$, which are in $\mathrm{mg} \cdot \mathrm{L}^{-1}$; ${ }^{\mathrm{b}} \mathrm{I}$ : indoor samples (I-HW); O: outdoor samples $(\mathrm{O}-\mathrm{HW})$; ${ }^{\mathrm{c}} \mathrm{Same}$ number for I-HW and O-HW; ${ }^{\mathrm{d}}$ Samples from several locations within the governorate; ${ }^{\mathrm{e}} \mathrm{All}$ of the household samples from the locations and governorates. NA $=$ not analyzed; $\mathrm{ND}=$ not detected. 


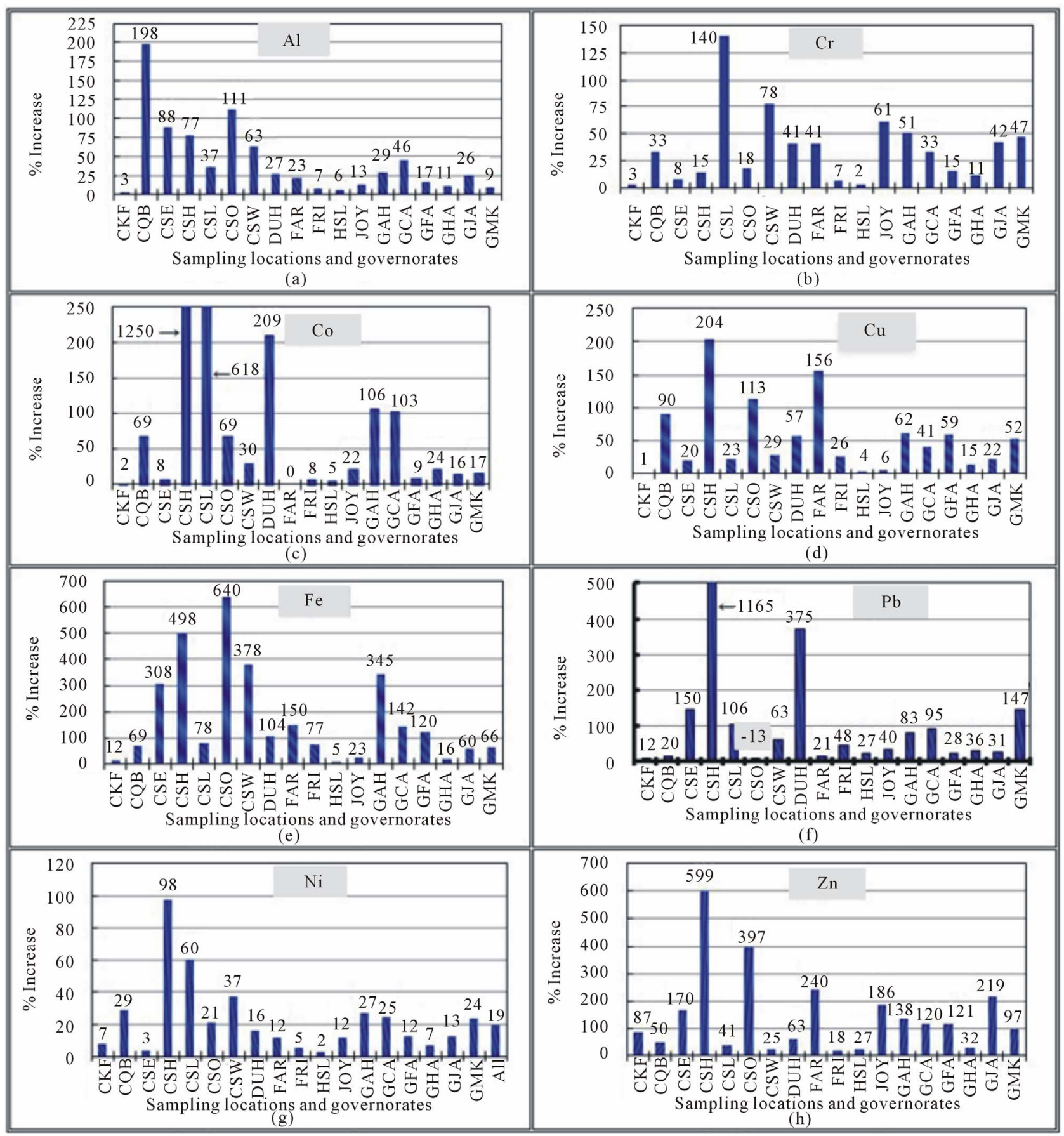

Figure 3. Variations in the increases of various trace elements in indoor household drinking water (I-HW) among sampling locations and governorates.

mean O-HW value $\left(33.5 \mu \mathrm{g} \cdot \mathrm{L}^{-1}\right)$, with a difference of only $3 \%$. The higher mean values of I-HW and O-HW samples may be attributed to the source water and the piping network in the area. Additionally, we can conclude that the increase of $\mathrm{Al}$ in the I-HW samples of the GCA governorate was the result of leaching from household utilities and piping at the 38 sampling locations, whereas the source water and the piping network were the source of $\mathrm{Al}$ in the I-HW and O-HW samples of the six sampling locations in the GMK governorate. The household utilities and piping contributed less of an $\mathrm{Al}$ increase (compared with CQB) in the I-HW samples of the CSO (111\%), CSE (88\%), CSH (77\%), and CSW (63\%) locations, with even lower increases at the CSL 
(37\%), DUH (27\%), and FAR (23\%) locations and much lower increases at the JOY (13\%), FRI (7\%), and HSL (6\%) locations.

The highest values of $\mathrm{Cr}\left(2.0 \mu \mathrm{g} \cdot \mathrm{L}^{-1}\right)$, Co $\left(2.2 \mu \mathrm{g} \cdot \mathrm{L}^{-1}\right)$, and $\mathrm{Pb}\left(2.2 \mu \mathrm{g} \cdot \mathrm{L}^{-1}\right)$ were found in the O-HW samples. The mean values of $\mathrm{Cr}, \mathrm{Co}$, and $\mathrm{Pb}$ in the I-HW and the $\mathrm{O}$-HW did not exceed $1.0 \mu \mathrm{g} \cdot \mathrm{L}^{-1}$, which is a fairly low level, at any location or governorate, with the following exceptions for the I-HW samples: 1) $\mathrm{Cr}$ in CSL (1.3 $\left.\mu \mathrm{g} \cdot \mathrm{L}^{-1}\right)$, JOY $\left(1.5 \mu \mathrm{g} \cdot \mathrm{L}^{-1}\right)$, and GJA $\left.\left(1.1 \mu \mathrm{g} \cdot \mathrm{L}^{-1}\right) ; 2\right)$ Co in $\operatorname{CSH}\left(8.1 \mu \mathrm{g} \cdot \mathrm{L}^{-1}\right)$, CSL $\left(4.1 \mu \mathrm{g} \cdot \mathrm{L}^{-1}\right)$, and GCA $(1.2$ $\left.\mu \mathrm{g} \cdot \mathrm{L}^{-1}\right)$; and 3) $\mathrm{Pb}$ in CSH $\left(6.3 \mu \mathrm{g} \cdot \mathrm{L}^{-1}\right)$ and GCA $(1.1$ $\left.\mu \mathrm{g} \cdot \mathrm{L}^{-1}\right)$. The lowest increase of $\mathrm{Cr}(2 \%)$ was found in HSL, whereas the highest increases were found in CSL and CSW (140\% and 78\%, respectively) (Figure 3(b)). The maximum $\mathrm{Cr}$ values in the I-HW and O-HW samples were 5.2 and $2.5 \mu \mathrm{g} \cdot \mathrm{L}^{-1}$, respectively, which is well below the WHO guideline of $50 \mu \mathrm{g} \cdot \mathrm{L}^{-1}[40]$. The increase in the Cr between the O-HW and I-HW samples may be the result of leaching from faucets and/or the metallic parts of household utilities [41]. There was no increase of Co in FAR, and the highest increases were found in CSH and CSL (1250\% and 618\%, respectively) (Figure 3(c)). There was a 13\% decrease in the level of $\mathrm{Pb}$ from the O-HW samples to the I-HW samples in CSO (Figure 3(f)), which is likely spurious because of the low levels of $\mathrm{Pb}$ detected. The highest $\mathrm{Pb}$ increases were found in CSH and DUH (1165\% and 375\%, respectively). The $\mathrm{Pb}$ in $\mathrm{CSH}$ exceeded the WHO guideline of 10 $\mu \mathrm{g} \cdot \mathrm{L}^{-1}[40]$. The Co levels in the I-HW samples of CSH and CSL did not pose any health concerns, despite the large increases in both. The highest recorded value was $24.3 \mu \mathrm{g} \cdot \mathrm{L}^{-1}$, and no guideline values for Co have been recommended by the WHO [40] or the US-EPA [42].

The leaching of $\mathrm{Cu}$ is strongly related to alkalinity and $\mathrm{pH}$. Decreasing $\mathrm{pH}$ and increasing alkalinity results in increased $\mathrm{Cu}$ leaching [43]. The highest and lowest increases in $\mathrm{Cu}$ were found at CSH and CKF (204\% and $1 \%$, respectively) among the locations and in GAH and GHA (62\% and $15 \%$, respectively) among the governorates (Figure 3(d)). The ranges of the mean values of $\mathrm{Cu}$ for the I-HW and O-HW samples were $32-129 \mu \mathrm{g} \cdot \mathrm{L}^{-1}$ and $17-55 \mu \mathrm{g} \cdot \mathrm{L}^{-1}$, respectively, within the locations and $40-70 \mu \mathrm{g} \cdot \mathrm{L}^{-1}$ and $26-44 \mu \mathrm{g} \cdot \mathrm{L}^{-1}$, respectively, within the governorates (Table 2). The highest mean value of $\mathrm{Cu}\left(129 \mu \mathrm{g} \cdot \mathrm{L}^{-1}\right.$, found in the I-HW sample collected at the FAR location) was probably the result of leaching from the household metallic utilities, specifically from $\mathrm{Cu}$ piping (Table 1). The solubility of $\mathrm{Cu}$ piping depends on the level of dissolved organics and on the aggressivity of the water $[44,45]$.

Galvanized iron, ductile iron, cast iron, and steel are iron-based materials that constitute the main sources of iron in the CP-DW in production and transportation equipment and piping or in household utilities. It has been reported that the leaching of Fe usually decreases with increasing $\mathrm{pH}$ and that corrosion rates decrease with increasing alkalinity at a fixed $\mathrm{pH}$ [46]. Among the elements in the 8-TE group, $\mathrm{Fe}$ had the highest increase (135\%) from the I-HW samples to the O-HW samples out of the 321 samples collected (Figure 2). This value represents the mean of the increases in all locations and governorates and ranges from $5 \%$ in HSL to $640 \%$ in CSO (Figure 3(e)). The HSL sampling location, which was one of the six apartments in a 3-story building with nonmetal utilities and a high rate of water consumption caused by a high population of residents, had the lowest $\mathrm{Fe}$ increase. The CSO location, which was a moderately old house with six occupants and galvanized iron utilities, had the highest increase of Fe. The CQB location had a much lower Fe increase (69\%) than the CSO (640\%), CSH (498\%), CSW (378\%), and CSE (308\%) locations but exhibited the highest mean I-HW and O-HW values $\left(576 \mu \mathrm{g} \cdot \mathrm{L}^{-1}\right.$, with a maximum of $1589 \mu \mathrm{g} \cdot \mathrm{L}^{-1}, 340 \mu \mathrm{g} \cdot \mathrm{L}^{-1}$, with a maximum of $987 \mu \mathrm{g} \cdot \mathrm{L}^{-1}$, respectively). The CSO, CSH, CSW, and CSE locations had mean I-HW and $\mathrm{O}-\mathrm{HW} \mathrm{Fe}$ values of 167 and $23 \mu \mathrm{g} \cdot \mathrm{L}^{-1}, 98$ and $16 \mu \mathrm{g} \cdot \mathrm{L}^{-1}$, 51 and $11 \mu \mathrm{g} \cdot \mathrm{L}^{-1}$, and 92 and $23 \mu \mathrm{g} \cdot \mathrm{L}^{-1}$, respectively, with maximum values of 388 and $59 \mu \mathrm{g} \cdot \mathrm{L}^{-1}, 134$ and 21 $\mu \mathrm{g} \cdot \mathrm{L}^{-1}, 72$ and $24 \mu \mathrm{g} \cdot \mathrm{L}^{-1}$, and 351 and $93 \mu \mathrm{g} \cdot \mathrm{L}^{-1}$, respectively. The CKF location had the lowest mean value for $\mathrm{Fe}$, with $9.5 \mu \mathrm{g} \cdot \mathrm{L}^{-1}$ in the I-HW samples and an increase of $12 \%$, which was slightly higher than that of the HSL location (5\% increase and $31 \mu \mathrm{g} \cdot \mathrm{L}^{-1}$ mean value) (Figure 3(e), Table 2). Among the governorates, GAH (with 16 sampling locations) had the highest increase in Fe between the O-HW and I-HW samples (345\%), with a mean I-HW value of $65 \mu \mathrm{g} \cdot \mathrm{L}^{-1}$, followed by GCA (with 38 sampling locations), with an increase of $142 \%$ and the highest mean value of $103 \mu \mathrm{g} \cdot \mathrm{L}^{-1}$. Again, the higher values in CQB compared with the other locations were caused by the corrosion of indoor piping and utilities (for I-HW samples) and the outdoor piping of the distribution network (for O-HW samples) (Table 1).

The most important source of $\mathrm{Ni}$ in drinking water is generally the passive leaching of ions from the surfaces of metallic piping, nickel-plated taps, tap valves, and metallic connectors for polypropylene piping rather than corrosion [41,47]. Because of the lower Ni content in the equipment used for water production and distribution as well as in household utilities compared with other metals, the mean level of $\mathrm{Ni}$ and the leaching from household utilities in all locations were significantly lower than those of $\mathrm{Al}, \mathrm{Cu}, \mathrm{Fe}$, and $\mathrm{Zn}$. The ranges of the mean values of Ni were $1.8-9.3 \mu \mathrm{g} \cdot \mathrm{L}^{-1}$ and $2.1-12.8 \mu \mathrm{g} \cdot \mathrm{L}^{-1}$ for the I-HW and O-HW samples, respectively. Figure 3(g) 
shows that the Ni increase ranged from $2 \%$ for HSL to $98 \%$ for CHS. The lowest mean values of $\mathrm{Ni}$ were found at DUH $\left(1.8\right.$ and $2.1 \mu \mathrm{g} \cdot \mathrm{L}^{-1}$ for I-HW and O-HW, respectively), whereas the highest values were found at the CSW location (9.3 and $12.8 \mu \mathrm{g} \cdot \mathrm{L}^{-1}$ for I-HW and O-HW, respectively). These results illustrate the role of water production equipment and the distribution network in the leaching of $\mathrm{Ni}$ in the $\mathrm{O}-\mathrm{HW}$, as DUH and CSW were completely fed by the Az-Zoor and Shuwaikh plants, respectively. The lowest mean values of $\mathrm{Ni}$ in the O-HW samples among governorates were found in GAH and GMK (2.3 $\mu \mathrm{g} \cdot \mathrm{L}^{-1}$ and $2.6 \mu \mathrm{g} \cdot \mathrm{L}^{-1}$, respectively), which are fed by the Az-Zoor plant, whereas the highest mean value was found in GCA $\left(6.7 \mu \mathrm{g} \cdot \mathrm{L}^{-1}\right)$, in which most of the 38 sampling locations are fed by the Shuaikh and Doha plants.

$\mathrm{Zn}$ is widely used for galvanizing steel and iron products as well as the production of corrosion-resistant alloys. These products are widely used in drinking water production utilities, distribution systems, and storage facilities. In this study, the average increase of $123 \%$ for $\mathrm{Zn}$ between the O-HW and I-HW samples from various locations and governorates was the second highest increase, after the increase in $\mathrm{Fe}$. The increase in $\mathrm{Zn}$ varied between 18\% (in FRI) and 599\% (in CSH), as illustrated in Figure 3(h). The increase of $\mathrm{Zn}$ in the CHS samples was 12 times higher than the increase in the CQB samples, even though the mean values of $\mathrm{Zn}$ in the I-HW samples of CQB and CSH were the highest out of all of the locations and were similar (231 and $194 \mu \mathrm{g} \cdot \mathrm{L}^{-1}$, respectively) (Table 2). This large difference in the increase of $\mathrm{Zn}$ between the two locations (CQB and CHS) was caused by higher leaching from the outdoor piping in CQB compared with CHS. The O-HW mean values were 154 and $28 \mu \mathrm{g} \mathrm{L}^{-1}$ for CQB and CHS, respectively (Table 2). CKF had the lowest mean values for the I-HW and $\mathrm{O}-\mathrm{HW}$ samples (16.3 and $8.7 \mu \mathrm{g} \cdot \mathrm{L}^{-1}$, respectively) with a fairly low increase of $\mathrm{Zn}(87 \%)$. Among the governorates, the highest and lowest increases of $\mathrm{Zn}$ were found in GJA (219\%, with nine locations) and GHA (32\%, with 11 locations). These variations among the locations and governorates were related to the degree of leaching from indoor utilities and outdoor piping (Table 1). Galvanized iron releases high levels of $\mathrm{Zn}$, with a small contribution from taps and other metallic parts [41].

Al-Mudhaf and Abu-Shady [15] reported that $\mathrm{Fe}, \mathrm{Pb}$, and $\mathrm{Ni}$ exceeded the US-EPA [42] and WHO [40] guidelines in $8.5 \%, 0.3 \%$, and $1 \%$ of the I-HW samples, respectively. In this study, only Fe was found to exceed the US-EPA guidelines, in $3 \%$ of the O-HW samples. This finding indicates that household utilities may cause health concerns for the consumers of drinking water in
Kuwait. The I-HW samples of the earlier study [15] and the O-HW samples in this study were collected at the same time and analyzed on the same day.

The water characteristics have been found to determine the types and amounts of TEs leached from piping and utilities [48]. Wagner [49] reported that the free $\mathrm{CO}_{2}$ content and $\mathrm{pH}$ of the water were primarily responsible for the corrosion of the piping. Ruckert and Sturzbecher [50] found that the lifetime of the pipe inner surface coating was $\mathrm{pH}$-dependent. In this study, no correlations were found between the variations of the mean $\mathrm{pH}$ levels at various sampling locations and governorates and the corresponding increases in any of the elements in the 8-TE group. This lack of correlation may be the result of a convergence among the mean $\mathrm{pH}$ values, with ranges of $7.53-7.71$ and $7.51-7.70$ for the I-HW and O-HW samples, respectively.

Maessen et al. [51] reported that the degree of leaching of some of the TEs was strongly related to some of the variable WQPs, such as conductivity, TDS, $\mathrm{Cl}^{-}$, $\mathrm{SO}_{4}^{2-}$, and $\mathrm{NO}_{3}^{-}$. In this study, no correlations were observed among increases in $\mathrm{Al}, \mathrm{Co}, \mathrm{Cr}, \mathrm{Pb}$, and $\mathrm{Ni}$ and conductivity, $\mathrm{Cl}^{-}$, or $\mathrm{SO}_{4}^{2-}$. The variations in the increases of $\mathrm{Cu}, \mathrm{Fe}$, and $\mathrm{Zn}$ with conductivity, residual $\mathrm{Cl}$, and $\mathrm{Cl}^{-}$are illustrated in Figure 4. The increases in $\mathrm{Cu}$, $\mathrm{Fe}$, and $\mathrm{Zn}$ were inversely proportional to the conductivity in most cases (Figures 4(a1), (b1) and (c1), respectively) and directly proportional to the values of $\mathrm{Cl}^{-}$ (Figures 4(a2), (b2) and (c2), respectively). The same pattern was observed for TDS, which is often positively correlated with the conductivity of water. The TDS (ppm) usually ranges from 0.5 to 1.0 times the conductivity [18]. It has been reported that the degree of corrosion of some TEs increases with an increase in residual chlorine $\mathrm{Cl}[52$, 53]. Viraraghavan et al. [32] and Reiber [53] reported that the residual $\mathrm{Cl}$ levels strongly affect the degree of leaching of $\mathrm{Cu}$, even at levels lower than $0.1 \mathrm{mg} \cdot \mathrm{L}^{-1}$. In this study, no definite correlations were observed between the mean values of residual $\mathrm{Cl}$ and the increases in $\mathrm{Cu}$ (Figure 4(a3)), Fe (Figure 4(b3)), or Zn (Figure 4(c3).

El Hosary and Gawish [54] reported that the corrosion rate of water piping was higher during the summer months than the winter months. Table 3 illustrates the mean values of various trace elements in the indoor (I-HW) and outdoor (O-HW) drinking water samples collected in the summer and in the winter from all sampling locations. In agreement with the finding of El Hosary and Gawish [54], Figure 5 shows that the increases of the mean values of all elements in the 8-TE group in the I-HW samples except for $\mathrm{Al}$ were higher in the summer than in the winter. Ni increased the least, with comparable increases between the O-HW samples 


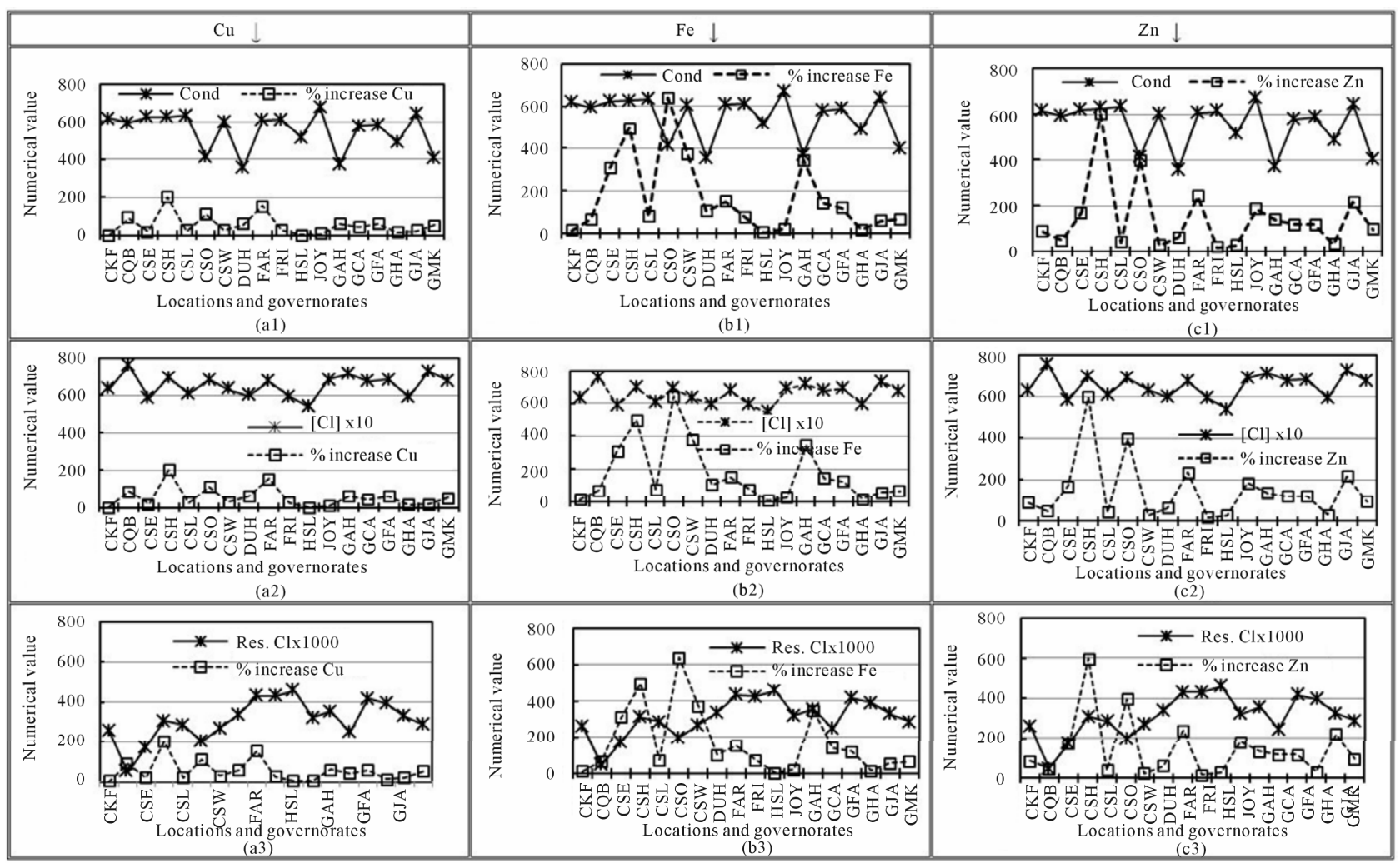

Figure 4. Variations in the increases of trace elements in indoor household drinking water (I-HW) with water quality parameters.

Table 3. Mean values of various trace elements of the indoor (I-HW) and outdoor (O-HW) drinking water samples collected in the summer and in the winter from all sampling locations.

\begin{tabular}{ccccc}
\hline \multirow{2}{*}{ Element } & \multicolumn{2}{c}{ Summer } & \multicolumn{2}{c}{ Winter } \\
\cline { 2 - 5 } & I-HW & O-HW & I-HW & O-HW \\
\hline Al & 13.0 & 10.8 & 10.9 & 8.10 \\
Cr & 0.70 & 0.53 & 0.66 & 0.52 \\
Co & 0.75 & 0.46 & 0.87 & 0.57 \\
Cu & 59.6 & 39.3 & 49.5 & 35.7 \\
Fe & 101 & 29.7 & 58.7 & 28.9 \\
Pb & 0.51 & 0.21 & 1.01 & 0.66 \\
Ni & 5.80 & 4.94 & 7.12 & 5.92 \\
Ti & 0.22 & 0.26 & 0.16 & 0.16 \\
V & 2.07 & 2.09 & 1.94 & 2.02 \\
Zn & 67.1 & 28.1 & 48.9 & 22.7 \\
\hline
\end{tabular}

and the I-HW samples in the summer and winter. Whereas Fe increased the most in the summer $(240 \%)$, followed by $\mathrm{Pb}(140 \%)$ and $\mathrm{Zn}(139 \%)$, the highest

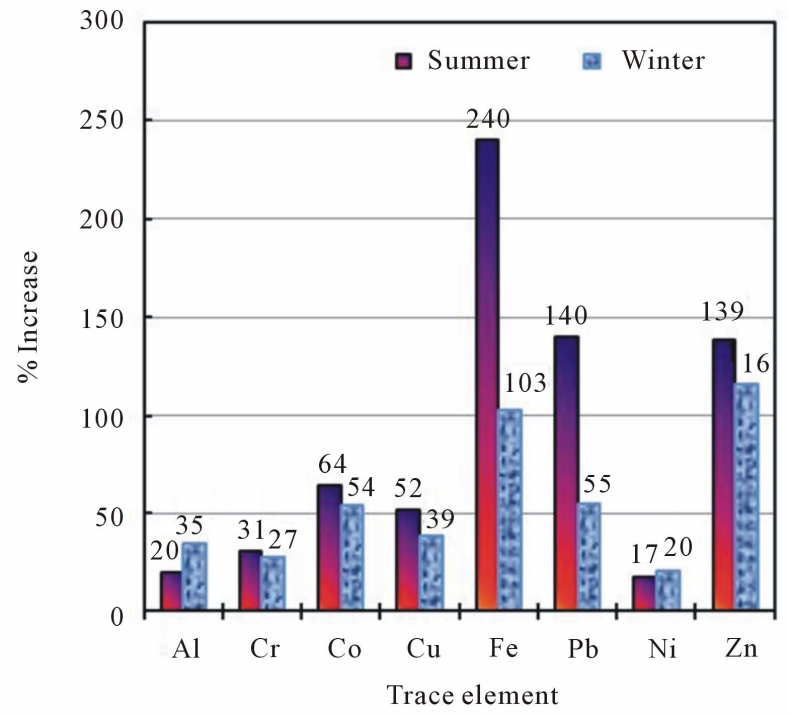

Figure 5. Variations in the increases of various trace elements in indoor household drinking water (I-HW) in the summer and in the winter.

increase in the summer relative to winter was observed for $\mathrm{Pb}(155 \%)$, followed by $\mathrm{Fe}(133 \%)$, and $\mathrm{Zn}$ exhibited the lowest increase $(20 \%)$. The increase of $\mathrm{Co}, \mathrm{Cu}$, and $\mathrm{Cr}$ in the I-HW samples were $64 \%, 52 \%$, and $31 \%$, re- 
spectively in the summer and $54 \%, 39 \%$, and $27 \%$, respectively, in the winter, with $19 \%, 33 \%$, and $15 \%$ increases, respectively, in the summer relative to the winter. Figure 6 shows the increases of the mean values of $\mathrm{Al}$, $\mathrm{Cr}, \mathrm{Cu}, \mathrm{Fe}$, and $\mathrm{Zn}$ in the summer relative to the winter for the I-HW and O-HW samples. The increases of $\mathrm{Cr}$, $\mathrm{Cu}, \mathrm{Fe}$, and $\mathrm{Zn}$ were higher in the I-HW samples than in the $\mathrm{O}-\mathrm{HW}$ samples, whereas the increases in $\mathrm{Al}$ were lower in the I-HW samples than in the O-HW samples. The highest increase was found in the I-HW samples of $\mathrm{Fe}(72 \%)$; this increase was significantly higher than that of the O-HW samples (3\%). The increases of $\mathrm{Co}, \mathrm{Pb}$, and Ni were slightly higher in the winter than in the summer for the I-HW and the O-HW samples. These observations indicate that household storage and plumbing utilities contribute to the increases in $\mathrm{Fe}$ through corrosion.

\section{Conclusions}

The corrosion of the metallic materials that come into contact with drinking water during its production and transportation via the general distribution network and through private household utilities was studied. Household desalinated drinking water samples collected from 99 locations and representing more than $95 \%$ of the residential areas in Kuwait were analyzed for 25 trace elements and water quality parameters. A comparison of household tap water at the consumption points (I-HW) with water in distribution networks just outside of buildings (O-HW) indicated that, 1) because of leaching from the household utilities, the mean concentrations of the elements in the 8-TE group ( $\mathrm{Al}, \mathrm{Cr}, \mathrm{Co}, \mathrm{Cu}, \mathrm{Fe}, \mathrm{Pb}, \mathrm{Ni}$,

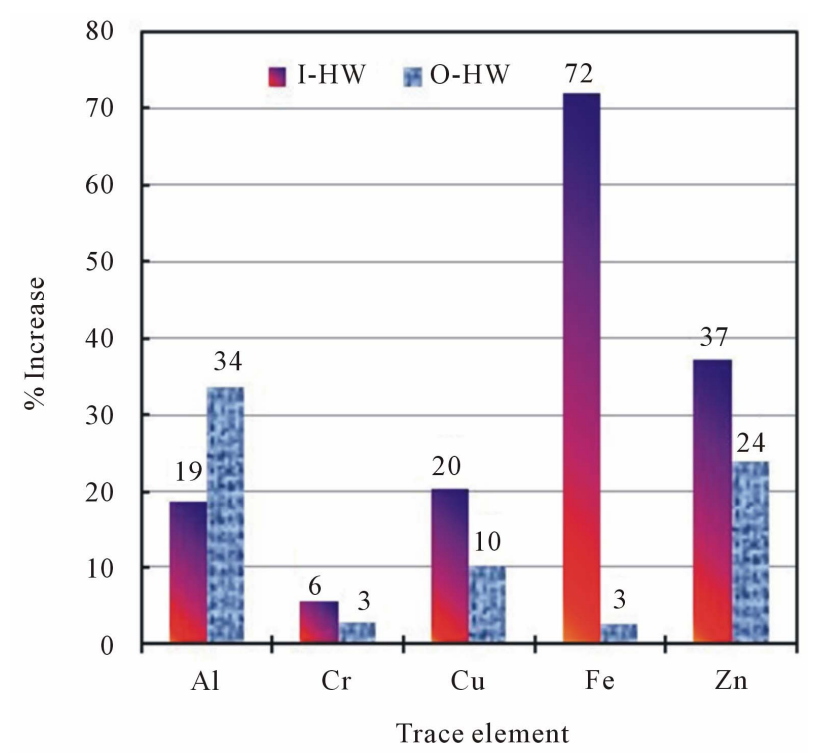

Figure 6. The increases of various trace elements in the summer relative to the winter for indoor (I-HW) and the outdoor (O-HW) household drinking water. and $\mathrm{Zn}$ ) were higher in the I-HW samples than in the O-HW samples at all sampling locations and in all governorates. Be was not detected in any of the samples, and there were no significant differences among the mean values of the remaining 16 TEs in the O-HW and I-HW samples. Additionally, 2) the highest increases were observed for $\mathrm{Fe}(135 \%)$ and $\mathrm{Zn}(123 \%)$, followed by $\mathrm{Pb}$ (69\%), Co (58\%), Cu (42\%), Cr (31\%), and $\mathrm{Al}(30 \%)$; the lowest increase was observed for Ni (19\%). Moreover, we found that 3 ) in most cases, the increases in $\mathrm{Cu}$, $\mathrm{Fe}$, and $\mathrm{Zn}$ were inversely proportional to the conductivity of the water and directly proportional to the concentration of $\mathrm{Cl}^{-}$. 4) $\mathrm{Fe}$ exceeded the US-EPA guideline in $3 \%$ of the O-HW samples and was the only element to exceed the guidelines in the O-HW samples, whereas Fe, $\mathrm{Pb}$, and $\mathrm{Ni}$ exceeded the US-EPA and WHO guidelines in $8.5 \%, 0.3 \%$, and $1 \%$ of the I-HW samples, respectively. Thus, leaching from household utilities may cause health concerns for the consumers of drinking water in Kuwait. Furthermore, 5) the mean values of all of the elements in the 8-TE group, with the exception of $\mathrm{Al}$, were higher in the I-HW samples in the summer than in the winter. The increase in $\mathrm{Fe}$ was the highest in the summer $(240 \%)$, and the largest difference (139\%) was observed between the summer and winter increases. 6) The complementary information regarding installed piping and utilities, premises, and desalination plants collected in this study reveal the current complexity of household systems and public water distribution networks operating in Kuwait.

The results of this study may prove useful for water production authorities and consumers in Kuwait and suggest the use of alternative new pipes with more resistant internal coatings and connecting techniques. Metallic materials that exhibit corrosion will be progressively replaced with new drinking-water-compatible polymeric and multilayer materials that have appeared in the market. In addition, new alloys will replace some materials currently used in the manufacturing of taps, valves, fittings, and connections to minimize the leaching of hazardous metals into household drinking water.

\section{Acknowledgements}

The authors acknowledge their gratitude for and appreciation of the Kuwait Foundation for the Advancement of Sciences (KFAS) for financial support for this study (grant \# 2001-1505-03) and the Public Authority for Applied Education and Training (PAAET) for partial financial support and guidance.

\section{REFERENCES}

[1] The World's Water 2008-2009, “The Biennial Report on 
Freshwater Resources," Island Press, Washington DC, 2009, p. 195.

http://www.worldwater.org/data20082009/Table1.pdf

[2] S. A. Imran, J. D. Dietz, G. Mutoti, W. Z. Xiao, J. S. Taylor and V. Desai, "Optimizing Source Water Blends for Corrosion and Residual Control in Distribution Systems," Journal of the American Water Works Association, Vol. 98, 2006, pp. 107-115.

[3] O. Lahav and L. Birnhack, "Quality Criteria for Desalinated Water Following Post Treatment," Desalination, Vol. 207, No. 1-3, 2007, pp. 286-303. doi:10.1016/i.desal.2006.05.022

[4] M. Rygaard, E. Arvin and P. J. Binning, "The Valuation of Water Quality: Effects of Mixing Different Drinking Water Qualities," Water Research, Vol. 43, No. 5, 2009, pp. 1207-1218. doi:10.1016/j.watres.2008.12.014

[5] World Health Organization (WHO), "Nutrients in Drinking Water. Water, Sanitation and Health Protection and the Human Environment," WHO, Geneva, 2005.

[6] O. A. Al-Khashman, "Study of Water Quality of Springs in Petra Region, Jordan: A Three-Year Follow-Up," Water Resource Management, Vol. 21, No. 7, 2007, pp. 1145-1163. doi:10.1007/s11269-006-9073-8

[7] S. I. Korfali and M. Jurdi, "Assessment of Domestic Water Quality: Case Study, Beirut, Lebanon," Environmental Monitoring and Assessment, Vol. 135, No. 1-3, 2007, pp. 241-251. doi:10.1007/s10661-007-9646-x

[8] S. Karavoltsosa, A. Sakellaria, N. Mihopoulosb, M. Dassenakisa and M. J. Scoullos, "Evaluation of the Quality of Drinking Water in Regions of Greece," Desalination, Vol. 224, No. 1-3, 2008, pp. 317-329. doi:10.1016/j.desal.2007.06.013

[9] J. Virkutyte and M. Sillanpää, "Chemical Evaluation of Potable Water in Eastern Qinghai Province, China, Human Health Aspects," Environment International, Vol. 32, No. 1, 2006, pp. 80-86. doi:10.1016/j.envint.2005.05.022

[10] U. I. Al-Saleh and I. Al-Doush, "Survey of Trace Elements in Household and Bottled Drinking Water Samples Collected in Riyadh, Saudi Arabia," Science of the Total Environment, Vol. 216, No. 3, 1998, pp. 181-192. doi:10.1016/S0048-9697(98)00137-5

[11] M. Ahmad and A. S. Bajahlan, "Quality Comparison of Tap Water vs. Bottled Water in the Industrial City of Yanbu (Saudi Arabia)," Environmental Monitoring and Assessment, Vol. 159, No. 1-4, 2009, pp. 1-14. doi:10.1007/s10661-008-0608-8

[12] K. M. Al-Fraij, M. K. Abd-El-Aleem and H. Al-Ajmy, "Comparative Study of Potable and Mineral Waters Available in the State of Kuwait," Desalination, Vol. 123, No. 2-3, 1999, pp. 253-264. doi:10.1016/S0011-9164(99)00081-8

[13] Z. S. Rizk, "Inorganic Chemicals in Domestic Water of the United Arab Emirates," Environmental Geochemistry and Health, Vol. 31, No. 1, 2009, pp. 27-45. doi:10.1007/s10653-008-9153-1

[14] A. O. Musaiger and Z. A. Khunji, "Chemical Quality of Drinking Water in Bahrain," Journal of the Royal Society of Health, Vol. 110, No. 3, 1990, pp. 104-105.

\section{doi:10.1177/146642409011000312}

[15] H. F. Al-Mudhaf and A. I. Abu-Shady, "Comparison of the Trace Element Contents in Bottled and Desalinated Household Drinking Water in Kuwait," CLEAN-Soil, Air, Water, Vol. 40, No. 9, 2012, pp. 986-1000. doi:10.1002/clen.201100618

[16] H. F. Al-Mudhaf, M. N. Al-Hayan and A. I. Abu-Shady, "Mineral Content of Bottled and Desalinated Household Drinking Water in Kuwait," CLEAN-Soil, Air, Water, Vol. 39, No. 12, 2011, pp. 1068-1080. doi:10.1002/clen.201100257

[17] H. F. Al-Mudhaf, A. I. Abu-Shady, M. I. Selim and F. A. Alsharifi, "Survey of Haloacetic Acids in Bottled and Household Drinking Water Produced from Thermal Desalination in Kuwait," Open Environmental Sciences, Vol. 3, 2009, pp. 66-78.

http://bentham.org/open/toenvirsj/openaccess2.htm

[18] H. F. Al-Mudhaf, M. I. Selim, A. M. Astel and A. I. AbuShady, "Spatial Variation of Haloacetic Acids in Indoor and Outdoor Desalinated Household Drinking Water in Kuwait," CLEAN-Soil, Air, Water, Vol. 39, No. 9, 2011, pp. 833-843. doi:10.1002/clen.201000459

[19] M. Y. Ali and J. P. Riley, "The Production of Brominated Methanes in Desalination Plants in Kuwait," Water Research, Vol. 23, No. 9, 1989, pp. 1099-1106. doi:10.1016/0043-1354(89)90153-X

[20] M.Y. Ali and J. P. Riley, "Distribution of Halomethanes in Potable Waters of Kuwait," Water Research, Vol. 24, No. 4, 1990, pp. 533-538. doi:10.1016/0043-1354(90)90240-7

[21] N. A. Latif, F. M. Al-Awadi and B. A. Colenutt, "Trihalomethanes (THMs) Formation in Multi-Stage Flash (MSF) Desalination Plants," Desalination, Vol. 74, 1990, pp. 205-226. doi:10.1016/0011-9164(89)85052-0

[22] H. F. Al-Mudhaf and A. I. Abu-Shady, "Trihalomethane Levels and Their Seasonal Variation in Drinking Water Produced from Thermal Desalination in Kuwait," Open Environmental Sciences, Vol. 2, 2008, pp. 88-100. http://bentham.org/open/toenvirsj/openaccess $2 . h t m$

[23] H. F. Al-Mudhaf, A. M. Astel, M. I. Selim and A. I. AbuShady, "Self-Organizing Map Approach in Assessment Spatiotemporal Variations of Trihalomethanes in Desalinated Drinking Water in Kuwait," Desalination, Vol. 252, No. 1-3, 2010, pp. 97-105. doi:10.1016/j.desal.2009.10.017

[24] H. F. Al-Mudhaf, F. A. Alsharifi and A. I. Abu-Shady, "Survey of Organic Contaminants in Household and Bottled Drinking Waters in Kuwait," Science of the Total Environment, Vol. 407, No. 5, 2009, pp. 1658-1668. doi:10.1016/j.scitotenv.2008.10.057

[25] K. M. Al-Fraij, A. Al-Odwani and M. K. Abd-El-Aleem, "Pollution of Drinking Water by Means of Transportations and Storage and the Proposed Strategies," WSTA, 5th Gulf Water Conference, Doha, Vol. 1, 24-28 March 2001, pp. 219-241.

[26] E. Veschetti, I. Achene, E. Ferretti, L. Lucentini, G. Citti and M. Ottaviani, "Migration of Trace Metals in Italian Drinking Waters from Distribution Networks," Toxico- 
logical and Environmental Chemistry, Vol. 92, No. 3, 2010, pp. 521-535. doi:10.1080/02772240903036139

[27] J. Taylor, Z. Tang, W. Xiao and S. Hong, "Monitoring of Distribution Water Qualities under Various Source Water Blending," Environmental Monitoring and Assessment, Vol. 117, No. 1-3, 2006, pp. 59-71. doi:10.1007/s10661-006-7672-8

[28] T. Viraraghavan, K. S. Subramanian and B. V. Rao, "Drinking Water at the Tap: Impact of Plumbing Materials on Water Quality," Journal of Environmental Science and Health, Part A, Vol. 31, No. 8, 1996, pp. 2005-2016. doi:10.1080/10934529609376470

[29] M. R. Sha Mansouri, H. P. Moghadas and G. Sahmskhorraabadi, "A Study of Leakage of Trace Metals from Corrosion of the Municipal Drinking Water Distribution System," Journal of Research in Medical Sciences, Vol. 8 , No. 3, 2003, pp. 1-6.

[30] Y. S. Tam and P. Elefsiniotis, "Corrosion Control in Water Supply Systems: Effect of $\mathrm{pH}$, Alkalinity, and Orthophosphate on Lead and Copper Leaching from Brass Plumbing," Journal of Environmental Science and Health, Part A, Vol. 44, No. 12, 2009, pp. 1251-1260. doi:10.1080/10934520903140009

[31] A. S. Hill, M. J. Friedman, S. H. Reiber, G. V. Korshin and R. L. Valentine, "Behavior of Trace Inorganic Contaminants in Drinking Water Distribution System," Journal of the American Water Works Association, Vol. 102, 2010, pp. 107-118.

[32] T. Viraraghavan, K. S. Subramanian and B. Venkata Rao, "Impact of Household Plumbing Fixtures on Drinking Water Quality-A Review," International Journal of Environmental Studies, Vol. 56, No. 5, 1999, pp. 717-743. doi: $10.1080 / 00207239908711234$

[33] I. A. Alam and M. Sadiq, "Metal Contamination of Drinking Water from Corrosion of Distribution Pipes," Environmental Pollution, Vol. 57, No. 2, 1989, pp. 167178. doi:10.1016/0269-7491(89)90008-0

[34] M. Payne, "Lead in Drinking Water," Canadian Medical Associaton Journal, Vol. 179, No. 3, 2008, pp. 253-254. doi:10.1503/cmaj.071483

[35] US-EPA, "Methods and Guidance for Analysis of Water, Office of Water," Washington DC, 20460, EPA 821-C-99004, 1999.

[36] WHO, "Nickel in Drinking Water," Geneva, 2005. WHO/SDE/WSH/05.08/55

[37] "European Union (EU) Directive Related with Quality of Water Intended for Human Consumption. 98/83/EC," Official Journal of the European Communities, Vol. L330, 1998, pp. 32-54.

[38] H. Baribeau, M. Prevost, R. Desjardins and P. Lafrance, "Changes in Chlorine and DOX Concentrations in Distribution Systems," Journal of the American Water Works Association, Vol. 93, 2001, pp. 18-32.

[39] American Water Works Association (AWWA), "Water Quality and Treatment," AWWA, USA, 1990.
[40] WHO, "Guidelines for Drinking-Water Quality, 3rd Edition, Recommendations," Incorporating 1st and 2nd Addenda, Vol. 1, WHO, Geneva, 2008.

[41] E. J. Hoekstra, V. Pedroni, R. Passarella, P. R. Trincherini and S. J. Eisenreich, "Elements in Tap Water, Part 3, Effect of Sample Volume and Stagnation Time on the Concentration of the Element," EUR 20672 EN/3, European Communities, 2004.

[42] US-EPA, "Drinking Water Standards and Health Advisories," EPA 822-R-04-005, Office of Water, US Environmental Protection Agency, Washington DC, 2004.

[43] J. Taylor, Z. Tang, W. Xiao and S. Hong, "Monitoring of Distribution Water Qualities under Various Source Water Blending," Environmental Monitoring and Assessment, Vol. 117, No. 1-3, 2006, pp. 59-71. doi:10.1007/s10661-006-7672-8

[44] M. Groenendijk and E. Coppens, "The Consequences of the New Parametric Value for Copper in Drinking Water," $\mathrm{H}_{2} \mathrm{O}$, Vol. 19, 2002, pp. 92-95.

[45] P. G. G. Slaats, E. J. M. Blokker and J. F. M. Versteegh, "Sampling Metals at the Tap: Analyses of Dutch Data over the Period 2004-2006," Proceedings of Cost Action 637 METEAU-Metals and Related Substances in Drinking Water, Antalya, Turkey, 2007, pp. 61-69.

[46] P. Sarin, J. A. Clement, V. L. Snoeyink and W. M. Kriven, "Iron Release from Corroded, Unlined Cast-Iron Pipe," Journal of the American Water Works Association, Vol. 95, 2003, pp. 85-96.

[47] W. Schwenk, "Nickel Transfer from Cr-Ni Stainless Steel Pipework into Potable Water," GWF Wasser Abwasser, Vol. 133, 1992, pp. 281-286.

[48] M. R. Schock and C. H. Neff, "Trace Metal Contamination from Brass Fittings," Journal of the American Water Works Association, Vol. 80, 1988, pp. 47-56.

[49] I. Wagner, "Internal Corrosion in Domestic Drinking Water Installations," Journal of Water Supply: Research and Technology-Aqua, Vol. 41, 1992, pp. 219-223.

[50] J. Ruckert and D. Sturzbecher, "Long Term Performance of Galvanized Steel Tubes in Drinking Water with Different $\mathrm{pH}-$ Value and Flow Velocity," Werkstoffe und Korrosion, Vol. 39, 1988, pp. 7-17.

[51] O. Maessen, B. Freedman and R. McCurdy, "Metal Mobilization in Home Well Water Systems in Nova Scotia," Journal of the American Water Works Association, Vol. 77, 1985, pp. 73-80.

[52] S. Reiber, "Galvanic Stimulation of Corrosion on LeadTin Solder-Sweated Joints," Journal of the American Water Works Association, Vol. 83, 1991, pp. 83-91.

[53] S. Reiber, "Copper Plumbing Surfaces: An Electrochemical Study," Journal of the American Water Works Association, Vol. 81, 1989, pp. 114-122.

[54] A. A. El Hosary and M. M. Gawish, "Corrosion and Corrosion Inhibition of Steel in Tap Water," Egyptian Journal of Chemistry, Vol. 23, 1980, pp. 67-70. 